\title{
Determination of the Lumped Constant for the $\alpha$-methyl-tryptophan Method for Estimating the Rate of Serotonin Synthesis
}

\author{
Author: Michael C. Vanier \\ Department of Neurology and Neurosurgery \\ McGill University \\ Montreil, Canada
}

Submitted: November, 1992

A Thesis submitted to the Faculty of Graduate Studies and Research

In Partial Fulfillment of the Requirements for the Degree of

Master of Science

(C) 1992

Michael C. Vanier

All rights reserved 
Lumped Constant Determination for the alpha-methyl-tryptophan method 


\section{Acknowledgements}

- Thanks to Dr. Mirko Diksic, my advisor, for extreme patience, cooperation, and much help all through this project.

- Thanks to Dr. Lucas Yamamoto, for providing the laboratory environment in which the experiments were done.

- Thanks to Janet Arts for technical assistance.

- Thanks to Drs. A. Takada and S. Yura for help with surgery.

- Thanks to Yannick Pouliot for help with the French translation of the abstract.

- Thanks to Dr. Scott Fraser for the use of the imaging facilities in his lab.

- Special thanks to Ms. Monique Ledermann for cheerfully handling the administrative details while I was 2500 miles away (and for delivering my thesis on time!). 


\section{Abstract/Abstrait}

The lumped constant (LC) for the $\alpha$ - $\left[{ }^{14} C\right]$ methyl-L-tryptophan method for the estimation of regional rates of serotonin synthesis in the rat brain was computed. The lumped constant represents a scaling factor with which the conversion of the rate of brain uptake of the tracer $\left(\alpha-\left[{ }^{14} C\right]\right.$ methyl-L-tryptophan) into the rate of serotonin synthesis is made. The method used involved independently computing the tissue uptake of the tracer and the tracee and calculating their ratio. The lumped constant was estimated as $0.40 \pm 0.15$. Similar experiments were performed using rats treated with the drug probenecid, which blocks the efflux of 5-HIAA (a metabolite of 5-HT) from the brain. This gave an estimated LC of $0.29 \pm 0.09$, which is significantly different from that obtained in control rats. However, when the experiments using probenecid were corrected for the difference in plasma tryptophan levels relative to control experiments, the LC was not significantly different $(0.48 \pm 0.15)$. Thus an LC of 0.40 is a reasonable estimate which can be used to estimate regional rates of 5 - $\mathrm{HT}$ synthesis. My experiments also provide data on the rate of tryptophan incorporation into brain proteins, as a side benefit of the methods used. 
La constante unie ("lumped constant", LC) a été evaluee pour la méthode du $\alpha-\left[{ }^{[4} C\right]$-méthyl-L-tryptophane pour l'estimation des taux régionaux de synthèse de sérotonine (5-HT) dans le cerveau du rat. La LC est un facteur de conversion employé dans la conversion du taux d'accumulation du traceur radio-actif $\alpha-\left[{ }^{14} C\right]$-méthylL-tryptophane en un taux de synthèse du 5-HT. La méthode employée utilise la computation indépendante des taux de accumulation du traceur par le cerveau et celui substrat indigène, suivi du calcul de leur ratio. La LC fut ainsi evaluée a $0.40 \pm$ 0.15. De plus, des expériences similaires furent effectuées sur des rats traités avec de la probenecid. Cette drogue bloque la perte hors du cerveau du 5-HIAA, un métabolite du 5-HT. La LC pour ces expériences fut de $0.29 \pm 0.09$, une valeur significativement différente de la valeur citée plus haut. Cependant, après une correction de la LC pour tenir compte de la difference des niveaux de tryptophane présent dans le plasma des animaux traités relativement à ceux des animaux témoins, j'ai obtenu une valeur de la LC proche de la valeur initiale, soit $0.48 \pm 0.15$. En conclusion, je crois qu'une valeur de LC de 0.40 constitue une approximation raisonnable qui pourrait être utiliser pour estimer des taux de synthèse régionaux de 5-HT. En plus de ces résultats, mes expériences ont aussi fournit dess données sur le taux $d$ 'incorporation de tryptophane dans les protéines du cerveau. 


\section{Contents}

$\begin{array}{lll}\text { Acknowledgements } & \text { ii }\end{array}$

Abstract $\quad$ iii

1 Introduction 1

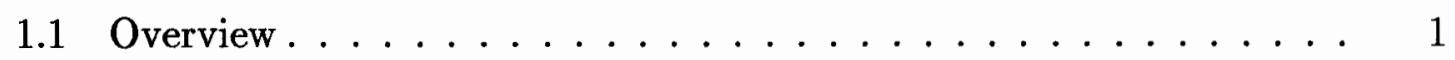

1.2 Serotonin and its metabolism ............... 4

1.3 Previous methods for measuring serotonin synthesis . . . . . . 8

1.3.1 Methods based on metabolic inhibitors ......... 8

1.3.2 Methods based on "turnover" measurements . . . . . . . 10

1.3.3 Methods using radioactive isotopes ........... 12

1.4 Need for a new method . . . . . . . . . . . . . 13

1.5 Original contributions to knowledge . . . . . . . . . . 15

2 Theory 16

2.1 The $\alpha$-methyl-tryptophan method . . . . . . . . . 16

2.1 .1 Background ......................... 16

2.1.2 Assumptions made in tracer-kinetic modelling . . . . . . . 19

2.1.3 The Full Operational Equation for the $\alpha$-[14 $C]$-methyl-L-tryptophan $\operatorname{method} \ldots \ldots \ldots \ldots 20 . \ldots \ldots$ 
2.1.4 The Linearized Approach using Time-Activity Plots . . . . . 22

2.2 The Operational Equation for the Rate of 5-HT Synthesis including $k_{e l} 24$

2.3 Determination of the Lumped Constant . . . . . . . . . . . 26

2.3.1 Determination of the Regional Rates of Tryptophan Incorporation into Proteins . . . . . . . . . . . . . . 28

2.4 Relationship of Rate Constants to Actual Enzyme Activities . . . . 29

2.5 Other Ways to Determine the Lumped Constant . . . . . . . . . 30

2.5 .1 Sokoloff's method . . . . . . . . . . . . . . . 30

2.5.2 Method of Phelps et. al. . . . . . . . . . . . . . 31

3 Materials and Methods $\quad 32$

3.1 Radiopharmaceuticals . . . . . . . . . . . . . . . . . 32

3.2 Surgical Procedures . . . . . . . . . . . . . . . . . 32

3.3 Probenecid treatment . . . . . . . . . . . . . . . 33

3.4 Plasma Tryptophan Determination $\ldots \ldots \ldots \ldots \ldots$

3.5 Data Analysis . . . . . . . . . . . . . . . 34

$\begin{array}{lll}4 & \text { Results } & 36\end{array}$

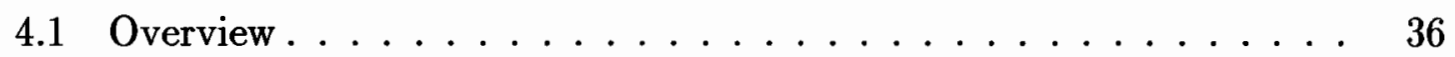

4.2 Results from previous work $\ldots \ldots \ldots \ldots \ldots$

4.3 Physiological parameters . . . . . . . . . . . . . 40

4.4 Results for serotonin synthesis . . . . . . . . . . . . . . 43

4.4.1 Sample time-activity plots . . . . . . . . . . 43

4.4.2 Rates of Serotonin Synthesis and Regional Lumped Constants 49

4.4.3 Determination of the Global Lumped Constant . . . . . . 54

4.5 Results for rates of tryptophan incorporation into protein . . . . . 58

4.5.1 Sample time-activity plots . . . . . . . . . . . . . 58

4.5.2 Rates of Tryptophan Incorporation into Protein . . . . . . 62 
5 Discussion $\quad 67$

5.1 Determination of the lumped constant . . . . . . . . . 67

5.2 Determination of the rate of tryptophan incorporation into protein . . 73

5.3 Possible future directions . . . . . . . . . . . . . 76

$\begin{array}{llr}6 & \text { Conclusions } & 78\end{array}$ 


\section{Chapter 1}

\section{Introduction}

\subsection{Overview}

Serotonin (5-hydroxytryptamine; 5-HT) is a monoaminergic neurotransmitter/ neuromodulator which is widely distributed in the mammalian CNS. Most serotonergic innervation of the mammalian brain derives from a group of nuclei lying along the midline of the pons and upper brainstem, the raphe nuclei [1] which in turn project their axons throughout the forebrain. 5-HT-containing neurons have also been located in other (non-raphe) nuclei such as the area postrema, the caudal locus coeruleus, and the interpeduncular nucleus [1]. Although the precise role of 5-HT in brain function is not understood, evidence exists showing an involvement of 5-HT in a large number of physiological, psychological and psychopathological conditions (reviewed in [2]). Alterations in 5-HT metabolism have been described in many pathological processes: affective disorders including both mania and depression [2, 3], obsessivecompulsive disorder $[2,4,5]$, suicidal behavior $[2,6,7]$, aggression $[8,9]$, eating disorders [10], alcoholism [2], panic disorder [2, 11], seasonal affective disorder [2], migraine [2,12], Alzheimer's disease [2, 13], and schizophrenia [2, 14]. In addition, 
many non-pathological states such as sleep $[2,15]$, pain $[2,16]$, memory [17], and sexual behavior $[2,18]$ have also been associated with alterations in serotonergic activity. Clearly, with so much evidence for the widespread involvement of 5-HT in a variety of brain processes, it would be desirable to have a method for estimating the rate of serotonin synthesis in different brain regions. This would allow more precise determinations of the role of 5 -HT in both normal and abnormal brain processes, and would also permit the assessment of the effects of drugs which interact with the serotonergic system on local serotonin metabolism. However, as described in greater detail below, until now there has been no reliable method for the determination of regional rates of 5-HT synthesis which had good spatial resolution. Work done in our laboratory $[19,20]$ provides the basis for such a method. This method is a quantitative autoradiographic method for the determination of the regional rates of 5-HT synthesis, and is based on a three-compartment model analogous to that described by Sokoloff et. al. for glucose [21]. The method uses a metabolic analogue of the 5-HT precursor tryptophan $\left(\alpha-\left[{ }^{14} C\right]\right.$-methyl-L-tryptophan) as a tracer for the synthetic pathway of 5-HT as described below.

The method described [19] requires the determination of a scaling constant (the "lumped constant" in Sokoloff's terminology) to account for the differences in the tracer's ( $\alpha-\left[{ }^{14} C\right]$-methyl-L-tryptophan) and the tracee's (L-tryptophan) behaviour in the brain. In the previous work $[19,20]$ the lumped constant, which can also be expressed as a function of several kinetic constants [21] was estimated by using published values of $K_{M}$ 's and $V_{m a x}$ 's (Michaelis-Menten constants for tryptophan hydroxylase, the rate-limiting enzyme for 5-HT synthesis), which at best can only give a rough idea of the true value since the results from which the values were taken varied considerably between different labs and different preparations. The objective of the work presented in this thesis is to determine the lumped constant directly in a number of individual brain regions, and to use these values to obtain a global estimate of the 
lumped constant. The method used also permits the determination of the rate of incorporation of tryptophan into brain protein, as a "bonus" of the procedure used.

In this introduction, I will begin by summarizing the relevant facts about the metabolism of serotonin. I will then discuss previously developed methods for determining the rate of serotonin synthesis and point out their drawbacks and the need for a new method. Finally, I will indicate what aspects of the work to be described in later sections constitutes an original contribution to knowledge about brain processes. 


\subsection{Serotonin and its metabolism}

The metabolism of serotonin has been thoroughly investigated (for a review, see $[1,22])$. The biochemical pathway of 5 -HT synthesis is diagrammed in figure 1 . I will describe the relevant steps below.

The amino acid L-tryptophan circulating in the plasma is transported by the large neutral amino acid (LNAA) transport protein across the blood-brain barrier (BBB) into the extracellular fluid. Two points must be made here. First, a large percentage (typically around $80-90 \%$ ) of plasma tryptophan is bound to plasma proteins such as albumin [23, 24]. Therefore, in the compartmental models of 5-HT synthesis, we have used the plasma concentration of free (unbound) tryptophan as the contents of the plasma compartment, under the assumption that only free tryptophan is directly related to the brain serotonin synthesis rate. Second, the LNAA transporter does not only transport tryptophan across the BBB but also several other amino acids including valine, isoleucine, leucine, phenylalanine, tyrosine, and methionine $[25,26$, 27]. Since these amino acids are competitive substrates for the LNAA transporter, the concentration of non-tryptophan amino acids in the plasma has a strong influence on the apparent $K_{M}$ of the LNAA transporter for tryptophan, and therefore the rate of tryptophan transport depends on the overall amino acid balance in the plasma.

L-tryptophan, once it has passed into the extracellular fluid, is transported in part into 5-HT-synthesizing neurons and is transformed into 5-hydroxy-L-tryptophan (5-HTP) by the enzyme tryptophan 5-hydroxylase (TH; E.C. 1.14.16.4). 'This step is generally considered to be the rate-limiting step for 5 -HT synthesis $[1,28]$ although other steps, such as tryptophan transport, may under certain circumstances (such as reduced blood flow) limit the overall rate of 5 -HT synthesis $[1,22]$. TH requires reduced tetrahydrobiopterin as a cofactor and molecular oxygen as a second substrate; thus, the level of oxygenation of the blood (and tissue) can also potentially affect the 
rate of 5 -HT synthesis; this has also been demonstrated experimentally $[1,29]$. With optimal concentrations of cofactors, the $K_{M}$ for tryptophan of TH is approximately 50 $\mu \mathrm{M}[1,22]$, which is roughly 5-7 times larger than the concentration of free tryptophan in the plasma and twice the concentration of tryptophan in the brain $(25 \mu \mathrm{M})$. Thus it appears that under normal conditions this enzyme is "unsaturated" with substrate, which is consistent with its postulated role as the rate-limiting step in 5-HT synthesis.

Once 5-HTP has been synthesized it is rapidly converted to 5 -HT by decarboxylation, which is accomplished by aromatic amino acid decarboxylase (AAAD; E.C. 4.1.1.28). AAAD is a pyridoxal- 5-phosphate-dependent enzyme which also is involved in the biosynthetic pathways for dopamine and norepinephrine [1,22]. This enzyme has a high affinity for 5 -HTP $\left(K_{M}=5 \mu M,[1]\right)$; this, along with the rapidity of its action and its high concentration in brain relative to the concentration of tryptophan hydroxylase [22] suggests that this step is not rate-limiting. This idea is also supported by the fact that 5-HTP is found in brain only in trace amounts [22].

5-HT is eventually catabolized by the enzyme monoamine oxidase (MAO; E.C. 1.4.3.4) to give the product 5-hydroxy-indoleacetaldehyde, which is rapidly reduced to 5-hydroxy-indoleacetic acid (5-HIAA). 5-HIAA is rapidly transported out of the brain by an acid transport system which can be blocked by the drug probenecid $[30,31]$. The tryptophan analogue $\alpha$-methyl-L-tryptophan, which was used in some of the experiments described below as a tracer for 5-HT synthesis, is transformed into $\alpha$-methyl-5-HT which is not a substrate for MAO [19], which makes it useful as a metabolic tracer as described in the Theory section.

In addition to the above metabolic steps, tryptophan is also incorporated into proteins in the brain. However, the tryptophan analogue $\alpha$-methyl-L-tryptophan is not incorporated into proteins to any significant extent $[19,32]$. In the case of my experiments using radiolabeled L-tryptophan (described below in the Results section), the incorporation of label into protein was subtracted from the total as 
described in the Theory and Materials and Methods sections. Tryptophan (but not $\alpha$-methyl tryptophan) is also a substrate for another enzyme, tryptophan pyrrolase, which eventually leads to the formation of quinolinic acid via the kynurenate pathway [33]. The proportion of brain tryptophan which is metabolized by this route in the brain is controversial, but most investigators believe that it is insignificant [34], so this pathway was not included in the compartmental models described below in the Theory section. 
Serotonin biosynthetic pathway:

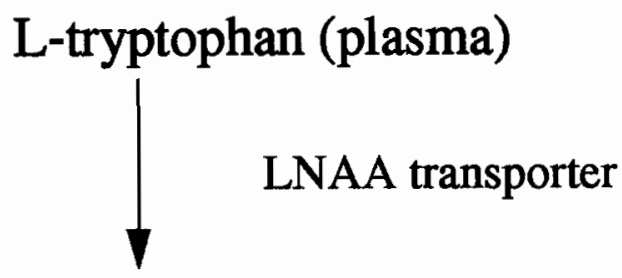

L-tryptophan (extracellular fluid)

$\downarrow \quad$ tryptophan transporter

L-tryptophan (intracellular)

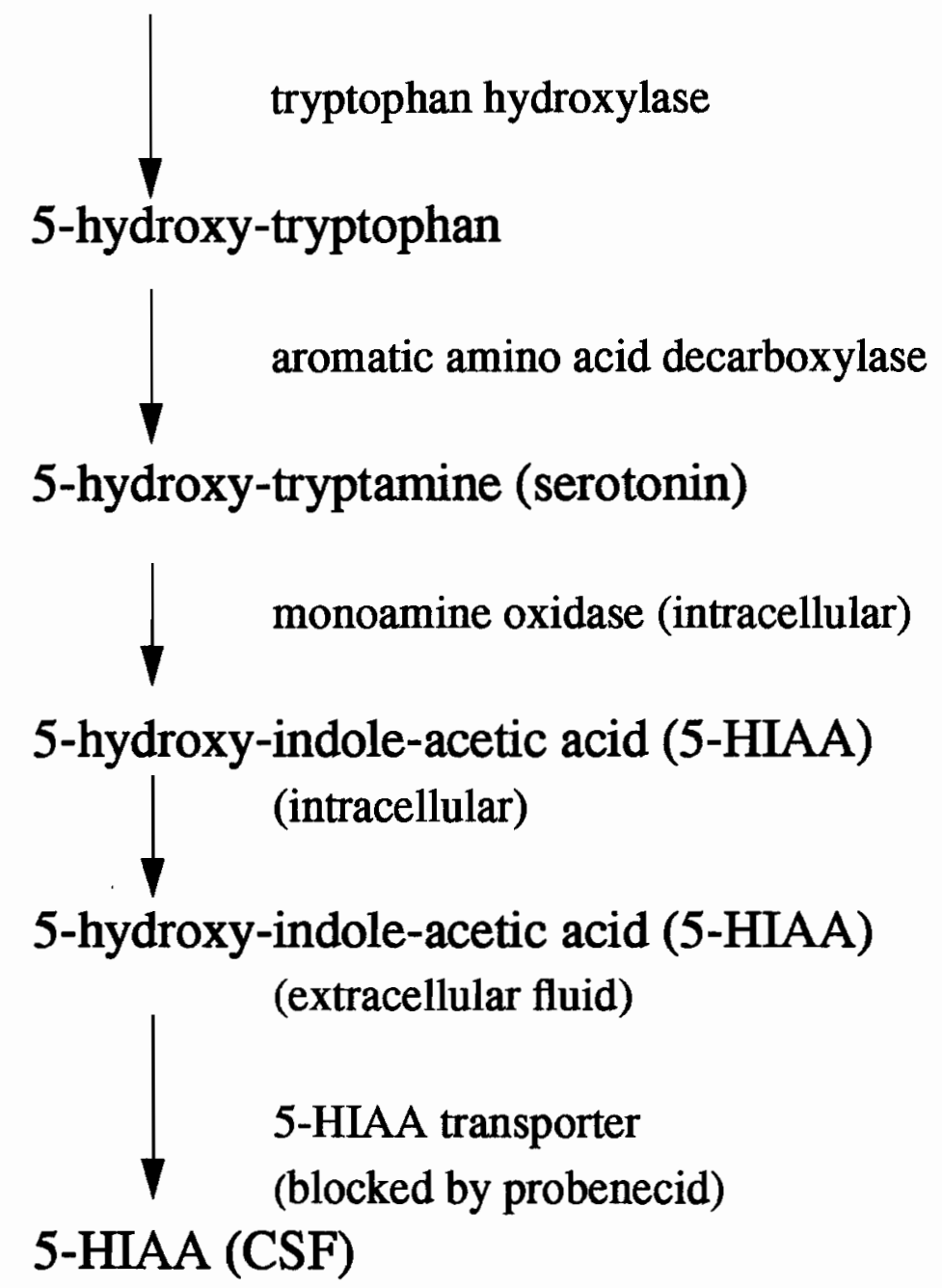

Figure 1. The biosynthetic pathway for serotonin. Note that deamination of 5-HT to 5-HIAA can also occur extracellularly after 5-HT release. 


\subsection{Previous methods for measuring serotonin syn- thesis}

A variety of methods have been used to measure the in vivo rate of 5 -HT synthesis. Below I will describe these methods and contrast them with the methods described in the Theory section.

\subsubsection{Methods based on metabolic inhibitors}

Probably the most widely used methods for measuring the in vivo rate of 5 -HT synthesis are based on the idea of inhibiting a critical enzyme in the biosynthetic or degradative pathway of 5-HT and observing the resulting buildup of the metabolite before the block. Examples of this include using drugs such as Ro4-4602 to block AAAD, leading to a buildup of 5-hydroxy-tryptophan [22], using MAO inhibitors such as pargyline to block 5-HT degradation, leading to a buildup of 5-HT [35], and using acid transport inhibitors such as probenecid to block 5-HIAA efflux to cerebrospinal fluid (CSF), leading to a buildup of 5 -HIAA [35, 36]. If the rate of 5 -HT synthesis is in a steady state and the relevant biochemical reactions obey first-order kinetics, we can use the following simplified kinetic scheme to describe 5-HT metabolism (from [35]):

$$
\text { (synthesis) } \longrightarrow \text { serotonin } \stackrel{r_{1}}{\longrightarrow} 5 \text {-HIAA } \stackrel{r_{2}}{\longrightarrow} \text { (excretion) }
$$

where $r_{1}$ and $r_{2}$ are first order rate constants with units of $\min ^{-1}$. Because of the steady-state conditions, the rate of 5-HT' formation will be equal to the rate of its 
degradation, which equals the rate of synthesis of 5-HIAA, which in turn also equals the rate of efflux of 5-HIAA (if units are in $n \mathrm{~mol} / \mathrm{g} / \mathrm{min}$ ). This rate is equal to [5HT]. $r_{1}$ and also to [5HIAA] $\cdot r_{2}$ (the terms in square brackets represent concentration in molar units). If we block any reaction, the concentration of the metabolite before the block will initially accumulate linearly while the concentration of the metabolite after the block will initially decrease monoexponentially [35] (this assumes that there is no feedback control of these reactions, such as end-product inhibition). Thus by measuring the concentrations of 5 -HT or 5-HIAA over time after blocking MAO or 5 -HIAA efflux we can estimate the rate constants $r_{1}$ or $r_{2}$ of the above reactions and use them along with measurements of the steady-state concentrations of metabolites to calculate the rate of 5 -HT synthesis.

One difficulty with this approach is that since the rate of 5-HT synthesis is estimated while the animal is being treated with drugs affecting the serotonergic system, the drugs may affect the metabolism of the system in unanticipated ways. For example, if the buildup of any one metabolite caused a significant amount of end-product inhibition, the estimated rate of 5-HT synthesis would be too small. On the other hand, unless this inhibition took effect very quickly one would be able to detect it by a change in the shape of the concentration-time curves (e.g. the concentration of the metabolite before the block would increase linearly and then the rate of increase would fall as the end-product inhibition took effect). This problem is circumvented somewhat by using only the initial slope of the concentration-time curve (or the initial monoexponential decline in product, if that method is used) to determine the rate constant(s). Tozer et al. [35] report that after MAO treatment the concentration of 5$\mathrm{HT}$ increased linearly for about 90 minutes and then approached an asymptote fairly abruptly. This would suggest that end-product inhibition of tryptophan hydroxylase by 5 -HT, while present, is not strong enough to cause difficulties for the method. A similar conclusion has been reached with methods involving the inhibition of efflux 
of 5-HIAA by probenecid [1] and with methods involving inhibition of AAAD [22].

Another difficulty lies in the fact that the drugs used can affect relevant quantities in unexpected ways. For example, probenecid, which blocks 5-HIAA efflux from the brain, also can increase the levels of free tryptophan in the plasma (see Results, below). This effect has also been seen with some MAO inhibitors [22]. Since the rate of 5-HT synthesis can be strongly affected by increasing the concentration of tryptophan (because the enzyme tryptophan hydroxylase is not normally saturated with tryptophan) this would mean that the estimates of the rate of 5-HT synthesis in methods using the above drugs would be overestimates. The autoradiographic methods described in the Theory section do not suffer from this drawback.

Finally, most of the papers using the above methods have only been able to estimate local rates of serotonin synthesis in the entire brain, or at best in very crude subdivisions (such as the brain stem and the rest of the brain [35]) due to the difficulty of reliably removing portions of the brain with good anatomical localization and which also are large enough to permit accurate determination of metabolite concentrations. One of the main advantages of the autoradiographic method described below is the high degree of spatial resolution achieved. With this method rates of 5 -HT synthesis can be estimated in all brain regions simultaneously; the resolution is only limited by the duration of exposure and the grain of the film.

\subsubsection{Methods based on "turnover" measurements}

Another quantity which is often used in the literature when discussing the metabolism of 5 -HT is the "turnover" of 5-HT. The term "turnover" has not always been used to refer to the same thing; what turnover usually means is the first order rate constant of 5-HT degradation ( $r_{1}$ above); if we assume that the rate constant for 5-HIAA efflux $\left(r_{2}\right)$ is constant throughout the brain (which is reasonable; see [35]) then we can write: 


$$
\begin{aligned}
r_{1} \cdot[5 H T] & =r_{2} \cdot[5 H I A A] \\
\frac{1}{r_{1}} & =\frac{[5 H T]}{[5 H I A A] \cdot r_{2}} \\
\frac{1}{r_{1}} & \propto \frac{[5 H T]}{[5 H I A A]}
\end{aligned}
$$

where $1 / r_{1}$ is the "turnover time" for 5 -HT, i.e. the time it would take a given quantity of 5 -HT to decay to $1 / e$ of the original value if 5 -HT synthesis is blocked. The symbol $\propto$ means "is proportional to". Thus by measuring the ratio of 5 -HT to 5-HIAA we get a figure which is proportional to the turnover time; this may be referred to as the "turnover ratio". The key point here is that this measurement tells us nothing about the rate of 5-HT synthesis; if the rate of 5-HT synthesis is increased or decreased but the constants $r_{1}$ and $r_{2}$ are unchanged then the ratio of 5-HT to 5-HIAA will not change (in the st eady state) even though the absolute values of the concentrations may change. Thus methods using turnover (at least this kind of turnover) do not address the issue of the absolute rate of 5-HT synthesis, although if one considers turnover to be the product of [5HT] and $r_{1}$ above (as is done in [37], for example) then this becomes equal to the rate of 5-HT degradation, which in the steady state equals the rate of 5 - $\mathrm{HT}$ synthesis. If one has estimated $r_{2}$ to a reasonable degree of accuracy and has determined that it is reasonably constant in different brain regions, then the turnover ratio can be used to estimate $r_{1}$, which then can be used along with $[5 H T]$ to calculate the rate of 5 -HT synthesis. The advantage of this method is that it does not require drug treatment of the animal which might perturb the steady state from the physiological values. The disadvantages are the 
need to have samples of brain large enough to do accurate quantitative analyses of the concentration of metabolites, which limits the anatomical resolution, as well as being much more laborious than autoradiographic methods.

\subsubsection{Methods using radioactive isotopes}

A variety of methods using radioactive compounds to trace the pathways for 5 -HT synthesis have been proposed [38, 39, 37, 40,41, 42]; most of these methods involve the injection of labelled tryptophan into the animal since, for instance, labelled 5-HT will not cross the blood-brain barrier in appreciable amounts [37]. The advantage of these methods is that since no pharmacological manipulations are required, the animal is in a state which is probably much closer to the real physiological steadystate. However, tryptophan is also a substrate for protein synthesis and participates in other metabolic reactions as well (see Serotonin and its metabolism, above), which complicates the models.

One approach $[37,40]$ involves the determination of the specific radioactivities of 5-HT in brain and tryptophan in plasma after infusion of tracer quantities of labelled tryptophan into the plasma at a constant rate. Given these quantities, the rate constant for 5-HT breakdown can be estimated by nonlinear curve fitting. This can then be used to obtain the rate of 5-HT synthesis. The difficulty with methods of this type is the need to measure both the concentration of tracer and the concentration of unlabelled tracee in the various brain regions. Determination of the concentration of the tracer is quite simple using autoradiography (see Materials and Methods) but chemical separation of metabolites is more laborious and requires a large enough tissue sample to permit accurate quantitation. In addition, each data point requires a terminal experiment (i.e. one rat must be used for each data point in the curve-fitting procedure). Nevertheless, methods of this sort have provided useful 
information on whole-brain rates of 5-HT synthesis which can be compared against the new autoradiographic methods described below in the Theory section.

Tracqui et. al. [41, 42] also used injections of labelled tryptophan, along with chemical separation of labelled tryptophan, 5-HT and 5-HIAA from animals killed at various times after injections. The approach taken was to fit the data to a number of compartmental models and fit the various parameters by nonlinear curve-fitting methods. Finally, a model was proposed which offered a good fit to the data with the smallest number of compartments. The final model proposed has four tryptophan compartments, two 5-HT compartments, and two 5-HIAA compartments. Although models such as this are useful for providing indirect evidence for the existence of different compartments in 5-HT metabolism, such a model, which tries to model all aspects of 5 -HT metabolism, is probably more complex than necessary if our goal is only to estimate the rate of 5-HT synthesis. In addition, it is difficult to use autoradiography to measure the radioactivities of three different metabolites simultaneously, although not necessarily impossible (e.g. with multiple tracers using different isotopes).

\subsection{Need for a new method}

Clearly, the autoradiographic method has advantages over the methods involving metabolic blockade of metabolites using drugs. However, the need to laboriously separate metabolites and estimate specific activities in different brain regions is much more complex than, for instance, direct autoradiographic methods which require only the knowledge of the time-course of plasma radioactivity and the concentration of the tracer at the end of the experiment, such as the 2-deoxyglucose method for estimation of the rate of glucose utilization [21]. The new method described below, like the 2-DG method, uses a metabolic analogue of the compound to be traced (in this case 5-HT) which has numerous advantages over the endogenous substrate. This compound, $\alpha$ - 
methyl-L-tryptophan, is metabolized into $\alpha$-methyl-5-HT, which as mentioned above is not a substrate for MAO degradation or protein synthesis [43] and so it accumulates in the final, "metabolic" compartment over time. This simplification of the kinetics allows us to calculate the local rates of 5-HT synthesis in rat brain with high spatial resolution if the following information is given (see Theory for details):

1. The average tissue radioactivities $(n C i / g)$ of all brain regions of interest at two times after injection of tracer ( 60 minutes and 150 minutes).

2. The plasma radioactivity concentration over time $(\mathrm{nCi} / \mathrm{ml})$ of all animals.

3. The concentration of free tryptophan in the plasma $(\mathrm{nmol} / \mathrm{ml})$ for all animals.

4. A scaling constant known as the "lumped constant", described below.

Given this information, the regional rate of 5-HT synthesis can be calculated. The "lumped constant" (LC) is a factor which accounts for metabolic differences and differences in tissue distribution volume: between the tracer and the tracee. This parameter does not have to be recalculated for each series of experiments, since it should only depend on enzyme-kinetic parameters such as the $K_{M}$ 's and $V_{\max }$ 's of the enzymes in the metabolic pathway with respect to the tracer and the tracee. However, if a treatment is given to the animal which alters these parameters, the LC may need to be re-evaluated.

Clearly this method is simpler and/or can provide higher spatial resolution than the methods described above. It does not require specific activity determinations of any metabolites in the pathway except for plasma tryptophan. The tracer has very simple kinetics which permits the use of a correspondingly simpler kinetic model, and the relevant parameters can be estimated with high reliability by a simple method, requiring only two independent measurements of brain radioactivity rather than a full time-course. As a result, it should be possible to use this method to obtain a great 
deal of new information about the rate of 5-HT synthesis in different brain regions under different conditions which was not previously available.

\subsection{Original contributions to knowledge}

The new method for the measurement of the rate of 5 -HT synthesis has been described elsewhere $[19,20]$. This work will be described in the Theory section as it is relevant to understanding the work $\mathrm{I}$ have done, and results obtained will be tabulated in the Results section. My project was to determine the lumped constant for this method, which had previously been estimated indirectly using data from the, literature. My experimental results and data analysis permit a direct determination of this parameter; in addition, my experiments also provide information on the rate of exogenous tryptophan incorporation into brain proteins. The lumped constant and its regional values for this method has not been described before and thus constitutes an original contribution to knowledge. In addition, the data presented here provides evidence that the drug probenecid acts to increase the rate of 5-HT synthesis solely by increasing the concentration of plasma free tryptophan, which has not been previously reported. It should be noted that I did not perform any of the experiments using $\alpha-\left[{ }^{14} C\right]$-methyl-L-tryptophan and none of the data in the two papers mentioned above was obtained by me. 


\section{Chapter 2}

\section{Theory}

\subsection{The $\alpha$-methyl-tryptophan method}

\subsubsection{Background}

The method we use for measuring the rate of serotonin synthesis is based upon a three-compartment tracer-kinetic model [19] (fig. 2), similar to the 2-deoxyglucose model of Sokoloff et. al. [21]. The tracer we use for measuring serotonin synthesis is $\alpha-\left[{ }^{14} C\right]$ methyl-L-tryptophan ( $\alpha$-MTrp), a tryptophan analogue whose metabolite, $\alpha-\left[{ }^{14} C\right]$ methyl-5-HT ( $\alpha$-M5HT), is not a substrate for MAO degradation [43] and thus is essentially trapped in the tissues, at least for the duration of the experiment. In the figure all variables and rate constants referring to the tracer or its metabolites are starred, while all those referring to the endogenous pathway are unstarred. The pathway for serotonin synthesis in the brain begins with the transfer of the amino acid L-tryptophan from the plasma across the blood-brain barrier (BBB) and into the extracellular fluid compartment. This step is represented in the model by the transfer coefficient $K_{1}\left(K_{1}^{*}\right)$, whose units are $\mathrm{ml} / \mathrm{g} / \mathrm{min}$. This parameter (technically a clearance) is actually the product of the plasma volume in $1 \mathrm{ml}$ of brain tissue mul- 
tiplied by the true first order rate constant $k_{1}\left(k_{1}^{*}\right)$, whose units are $\min ^{-1} . K_{1}$ is used because the concentration of L-tryptophan measured in the plasma compartment is in units of $\mathrm{nmol} / \mathrm{ml}$ and not $\mathrm{nmol} / \mathrm{g}$, as it is in the tissue measurements. Transfer of the tracer from the extracellular pool back across the BBB is represented by $k_{2}$ $\left(k_{2}^{*}\right)$, with units of $\mathrm{min}^{-1}$. The transfer of Trp or $\alpha$-MTrp into an irreversible compartment (presumably representing the conversion of tryptophan (or $\alpha$-MTrp) into 5 -HT (or $\alpha$-M5-HT) by tryptophan hydroxylase, although other possibilities exist) is represented by the rate constant $k_{3}\left(k_{3}^{*}\right)$. Note that there is no direct evidence for $k_{3}$ representing the rate of conversion of L-tryptophan to $5-\mathrm{HT}$, although it is the most reasonable hypothesis given the known metabolism of tryptophan in the brain. $\alpha$-M5-HT (or possibly $\alpha$-MTrp sequestered in an irreversible compartment, for example) is trapped in the tissues and accumulates over the period of the experiment. In contrast, 5-HT is a substrate for MAO degradation to 5-HIAA (fig. 1). The $\alpha$-methyl-tryptophan method thus uses a tracer which has an essentially non-metabolizable end product to simplify the kinetics of synthesis of the tracee in much the same way as the 2-deoxyglucose method for determining the rate of glucose synthesis [21]. In such methods, a scaling factor known as the lumped constant must be calculated to normalize for the differences between the tracer and the tracee (in terms of enzyme affinities, rates of transport etc.) In this chapter I will describe the main assumptions used in tracer-kinetic modelling, followed by two different methods by which one can measure the rate of serotonin synthesis, and finally a method by which the lumped constant can be calculated. 


\section{Serotonin Synthesis: Three-Compartment Model}

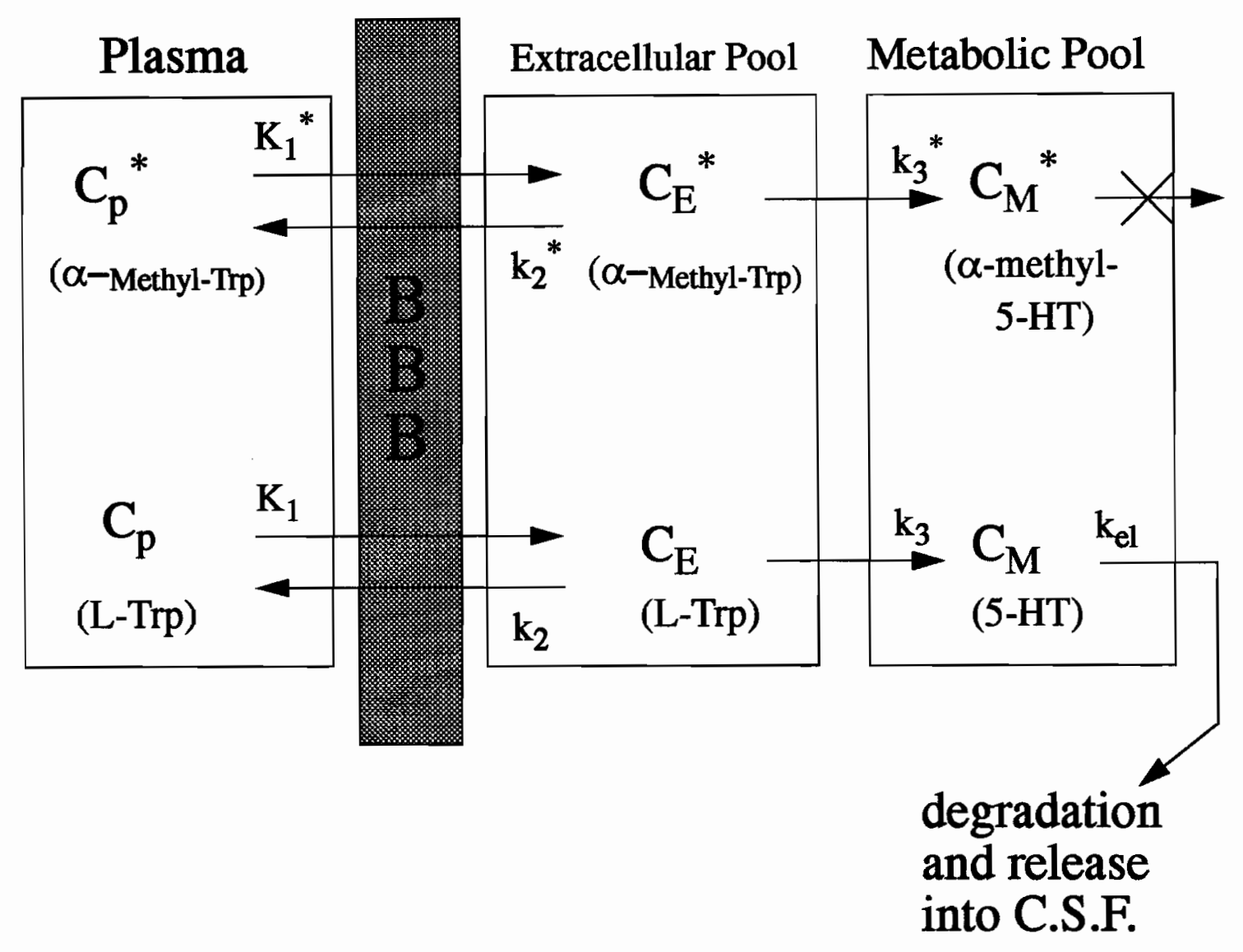

Figure 2: Three-compartment model for serotonin synthesis. Starred variables represent the radioactive tracer ${ }^{14} \mathrm{C}$ - $\alpha$-methyl-tryptophan or its metabolites. Note that the conversion of tryptophan (or $\alpha$-methyl-Trp) to 5-HT (or $\alpha$-methyl5-HT) actually consists of two steps:

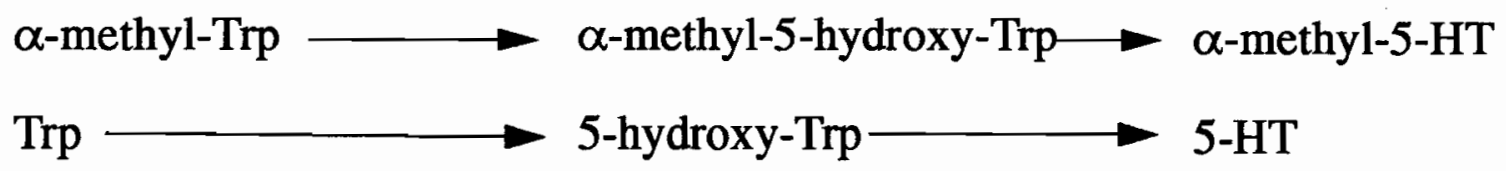

But conversion of $\alpha$-methyl-5-hydroxy-Trp to $\alpha$-methyl-5-HT or 5-hydroxy-Trp to 5 -HT is very rapid (not rate-limiting). 


\subsubsection{Assumptions made in tracer-kinetic modelling}

The primary assumption made in tracer-kinetic modelling is that the system is in a steady-state with respect to the tracee (i.e. in this case the rate of serotonin synthesis and the concentrations of all precursors and metabolites are in a steady-state). All experiments were performed at roughly the same time of day, to minimize the effects of diurnal variations in the overall rate of serotonin synthesis. In addition, all rats were fasted for $\sim 18$ hours before the experiments, which reduces the variation in plasma tryptophan levels caused by food digestion. It is also necessary to use a small enough mass of tracer so that the concentration of tracer is small with respect to the tracee (or else the system can be displaced from the steady-state during tracer infusion).

The "compartments" which are postulated in the three-compartment model do not represent actual physiological spaces, but are assumed to represent the net effect of the rate-limiting processes in the metabolic system of interest. Thus the "extracellular" compartment includes all tracer which has crossed the blood-brain barrier but has not been further metabolized. Clearly, this will include both extracellular and intracellular components as well as compartments representing different cell types; however, as long as the transfer of tracer between extra- and intracellular components is reversible (with the exception of the one irreversible compartment), which is to be expected, the fundamental equation of the method ( 2.7 below) can be shown to be valid [44]; this is true regardless of the number of compartments through which the tracer moves reversibly.

The "plasma" compartment would ideally consist of the concentration of tracer in the brain capillaries. Since this was not directly accessible to us, we have approximated this compartment by the concentration of tracer in the arterial blood, which was measured by taking blood samples from the femoral artery. If the fractional 
clearance of tracer from the plasma while passing through the brain is low, this will be a reasonable assumption since the difference between arterial plasma tryptophan levels and brain capillary tryptophan levels will be small.

We also assume that the equations which model the transfer of tracer from one compartment to another can be written using first-order rate constants. If this is not true, the resulting equations become nonlinear and analytically intractable. If the system is in fact in a steady-state, then only the tracer and its metabolites show any appreciable variation in concentration with time. Thus, even though a particular enzyme may require various substrates and cofactors, only one of them (representing the tracer or its metabolites) has a varying concentration, so the enzyme effectively can be treated as if it has only one substrate.

\subsubsection{The Full Operational Equation for the $\alpha-\left[{ }^{14} C\right]$-methyl- L-tryptophan method}

The fundamental rate equations for the $\alpha$-methyl-tryptophan method, are the following:

$$
\begin{aligned}
\frac{d C_{E}^{*}(t)}{d t} & =K_{1}^{*} \cdot f_{f}^{*} \cdot C_{p}^{*}(t)-\left(k_{2}^{*}+k_{3}^{*}\right) \cdot C_{E}^{*}(t) \\
\frac{d C_{M}^{*}(t)}{d t} & =k_{3}^{*} \cdot C_{E}^{*}(t) \\
C_{I}^{*}(t) & =C_{M}^{*}(t)+C_{E}^{*}(t)
\end{aligned}
$$

$C_{E}^{*}(t)$ represents the amount of (radioactive) $\alpha$-methyl-tryptophan in the extracellular compartment $(n C i / g) . C_{M}^{*}(t)$ represents the amount of $\alpha$-methyl-tryptophan in 
the metabolic compartment $(n C i / g) . C_{I}^{*}(t)$ represents the total tissue radioactivity $(n C i / g) . C_{p}^{*}(t)$ represents total plasma radioactivity $(n C i / m l) . k_{2}^{*}$ and $k_{3}^{*}$ are rate constants $\left(\mathrm{min}^{-1}\right)$ representing the transfer of the tracer from the extracellular compartment to the plasma compartment or the metabolic compartment, respectively. $K_{1}^{*}$ is a rate constant (clearance) $(\mathrm{ml} / \mathrm{g} / \mathrm{min})$ which represents transfer of the tracer across the BBB into the extracellular compartment. $f_{f}^{*}$ is the fraction of free (unbound) tracer in plasma, which is necessary since the a large proportion of tryptophan (and $\alpha$-methyl tryptophan) in plasma is bound to plasma protein [23, 24].

These equations can be solved using Laplace transforms to yield the following operational equation for the rate of serotonin synthesis [19]:

$$
R=\frac{C_{p}}{L C} \frac{\left[C_{I}^{*}(T)-f_{f}^{*} \cdot K_{1}^{*} \cdot \exp \left(-\left(k_{2}^{*}+k_{3}^{*}\right) \cdot t\right)\right] \otimes C_{p}^{*}(t)}{f_{f}^{*} \cdot\left[1-\exp \left(-\left(k_{2}^{*}+k_{3}^{*}\right) \cdot t\right)\right] \otimes C_{p}^{*}(t)}
$$

$\mathrm{LC}$ represents the lumped constant, a term which is a combination of enzyme-kinetic rate constants and other terms [21] as follows:

$$
L C=\frac{V_{\max }^{*} K_{M}}{V_{\max } K_{M}^{*}} \cdot \lambda
$$

where $V_{m a x}$ and $K_{M}$ represent Michaelis-Menten kinetic constants (with asterisks for tracer and without for tracee) and $\lambda$ equals the ratio of the volumes of distribution for the tracer and tracee $\left(\lambda=\left[K_{1}^{*} /\left(k_{2}^{*}+k_{3}^{*}\right)\right] /\left[K_{1} /\left(k_{2}+k_{3}\right)\right]\right.$, where rate constants without asterisks are those of unlabelled tryptophan).

This approach has a number of drawbacks, among them the fact that estimates of the individual rate constants tend to have large errors, and that a large fraction of $\alpha-\left[{ }^{14} C\right]$ methyl-L-tryptophan is in the precursor pool [19], requiring experiments of long duration to give a sufficiently large amount of metabolized tracer relative to the size of the precursor pool (or else the numerator of the operational equation will have a very large uncertainty since we are subtracting two numbers of similar magnitudes). 
These difficulties are largely circumvented in the method described next.

\subsubsection{The Linearized Approach using Time-Activity Plots}

In the above method, the individual rate constants must be determined by a nonlinear least-squares method, which may give large errors for the individual rate constants. Alternatively, one may calculate the net unidirectional influx constant $K^{*}$ :

$$
K^{*}=\frac{K_{1}^{*} \cdot k_{3}^{*}}{k_{2}^{*}+k_{3}^{*}}
$$

The constant $K^{*}$ can be calculated in a different way which does not require knowledge of the individual rate constants. Assuming that the system is governed by linear, firstorder kinetic equations, has a reversible transfer of tracer ( $\alpha$-MTrp in our case) across the $\mathrm{BBB}$, and has an irreversible final metabolic step, all of which are true in our system with $\alpha$-methyl-tryptophan as tracer, then the following equation can be shown to apply $[44,45]$ :

$$
C_{I}^{*}(T)=K^{*} \cdot \int_{0}^{T} C_{p}^{*} d t+V_{a p p} \cdot C_{p}^{*}(T)
$$

However, in our system, $C_{p}^{*}(T)$, which is the total plasma radioactivity $(n m o l / m l)$, must be replaced by $C_{p}^{*}(T) \cdot f_{f}$, where $f_{f}$ is the fraction of unbound tryptophan (or $\alpha$-methyl-tryptophan, if this is used as a tracer) calculated separately in each experiment as described above. We may also define

$$
\Theta(T)=\frac{\int_{0}^{T} C_{p}^{*}(t) d t}{C_{p}^{*}(T)}
$$

and thus we have:

$$
\frac{C_{I}^{*}(T)}{C_{p}^{*}(T) \cdot f_{f}}=K^{*} \cdot \Theta(T)+V_{a p p}
$$


where $V_{a p p}$ represents the apparent steady-state volume of distribution of the tracer $(m l / g)$ and $T$ is the duration of the experiment (e.g. $C_{I}^{*}(T)$ represents the tissue radioactivity at the end of the experiment). $\Theta(T)$ is the "exposure time": the ratio of the integral of the plasma radioactivity curve to the final plasma radioactivity value given at kill time. This represents the time that the tissue would have to be exposed to a constant plasma input of tracer (equal to the final value $C_{p}^{*}(T) \cdot f_{f}$ ) with the same time integral to give the same resultant tissue radioactivity. By taking samples of plasma throughout the experiment, we can measure $C_{p}^{*}(T)$ and calculate the time integral of the plasma curve using a nonlinear curve-fitting routine. From this, $\Theta(T)$ can be calculated. $C_{I}^{*}(T)$ can be calculated from autoradiograms of brain slices as described in Materials and Methods. In order to estimate $K^{*}$, we have to perform a number of different experiments of different durations and graph $\frac{C_{I}^{*}(T)}{C_{p}^{*}(T) \cdot f_{f}}$ vs. $\Theta(T)$. It has been shown $[44,45]$ that such "time-activity" plots will asymptote to a straight line if the assumptions given above are met. The slope of this line is $K^{*}$.

Once $K^{*}$ has been calculated, it can be shown $[19,20,46]$ that the rate of serotonin synthesis is equal to:

$$
R=\frac{C_{p}}{L C} \cdot K^{*}=\frac{K^{C_{p}}}{L C}
$$

$C_{p}$ was calculated by averaging the plasma free tryptophan levels for the group of experimental subjects. $K^{C p}$ refers to the product of $K^{*}$ and the averaged $C_{p}$. For experiments involving $\mathrm{L}-\left[{ }^{14} C\right]$-tryptophan as a tracer, this will be equal to the rate of 5-HT synthesis, whereas for experiments using $\alpha-\left[{ }^{14} C\right]$-methyl-L-tryptophan it will not be. In order to use this equation, the lumped constant must be known. Calculation of the lumped constant will be discussed in the next section. It might appear that in order to use the above equation, one would have to do a full time-series for each treatment of interest. However, a more economical way to calculate the rate 
of serotonin synthesis uses only two time points, both of which are selected to be within the linear region of tracer accumulation but which are separated enough to permit a reasonable estimate of $K^{*}$ given that several samples are available for each of the two time points [20].

Using rats which have been fasted for $\sim 18$ hours makes the plasma amino acid concentrations relatively constant. This is important because the ratio of plasma tryptophan to other amino acids using the same transport enzyme (the large neutral amino acid (LNAA) carrier) is a critical determinant of brain tryptophan concentration $[23,24,47]$.

\subsection{The Operational Equation for the Rate of 5- HT Synthesis including $k_{e l}$}

The above methods are adequate when the transfer of tracer into the "metabolic" compartment is terminal i.e. when there is essentially no tracer efflux from the brain after it enters the metabolic compartment. This is true for experiments using $\alpha$ - $\left[{ }^{14} C\right]$ methyl-L-tryptophan as a tracer, since $\alpha$-methyl-5-HT is not a substrate for $\mathrm{MAO}$ as mentioned above, so that it accumulates in the metabolic compartment. It is true for experiments using $\mathrm{L}-\left[{ }^{14} C\right]$-tryptophan as a tracer with rats treated with probenecid, even though in this case radiolabelled 5-HT can be converted to 5-HIAA. This 5-HIAA must remain in the brain tissue since probenecid blocks its efflux into the CSF, so the movement of radioactivity from 5-HT to 5-HIAA will not affect the total tissue radioactivity in this case. The linear time-activity method described above is also appropriate for determining the rate of tryptophan incorporation into protein (see below) because once radioactivity enters the pool of brain proteins it will not leave at a significant rate, since the pool of brain protein is so large. 
The above methods, however, are not appropriate when estimating $K^{*}$ values for experiments using $\mathrm{L}-\left[{ }^{14} C\right]$-tryptophan as a tracer with rats not treated with probenecid, because in this case significant losses of radioactivity from the metabolic compartment to the CSF in the form of 5-HIAA can occur. This is represented in figure 2 by $k_{e l}$, the elimination constant corresponding to losses of label from the metabolic compartment. In order to incorporate this constant into a method which will permit us to determine $K^{*}$, we must rewrite the basic rate equations as follows:

$$
\begin{aligned}
\frac{d C_{E}^{*}(t)}{d t} & =K_{1}^{*} \cdot f_{f}^{*} \cdot C_{p}^{*}(t)-\left(k_{2}^{*}+k_{3}^{*}\right) \cdot C_{E}^{*}(t) \\
\frac{d C_{M}^{*}(t)}{d t} & =k_{3}^{*} \cdot C_{E}^{*}(t)-k_{e l} \cdot C_{M} \\
C_{I}^{*}(t) & =C_{M}^{*}(t)+C_{E}^{*}(t)
\end{aligned}
$$

These equations can be solved by Laplace transform methods to yield the following operational equations:

$$
\begin{gathered}
C_{I}^{*}(T)=V 1 \cdot \int_{0}^{T} \exp \left(-k_{e l}(T-t)\right) C_{p} \cdot f_{f}^{*} d t+V 2 \cdot \int_{0}^{T} \exp \left(-\left(k_{2}^{*}+k_{3}^{*}\right)(T-t)\right) C_{p} \cdot f_{f}^{*} d t \\
\frac{C_{I}^{*}(T)}{C_{p}^{*} \cdot f_{f}^{*}}=\frac{V 1}{C_{p}^{*} \cdot f_{f}^{*}} \cdot \int_{0}^{T} \exp \left(-k_{e l}(T-t)\right) C_{p} \cdot f_{f}^{*} d t+\frac{V 2}{C_{p}^{*} \cdot f_{f}} \cdot \int_{0}^{T} \exp \left(-\left(k_{2}^{*}+k_{3}^{*}\right)(T-t)\right) C_{p} \cdot f_{f}^{*} d t
\end{gathered}
$$

where

$$
V 1=\frac{K_{1}^{*} \cdot k_{3}^{*}}{k_{2}^{*}+k_{3}^{*}-k_{e l}}=\frac{K^{*}}{1-\frac{k_{e l}}{k_{2}^{*}+k_{3}^{*}}}
$$




$$
V 2=\frac{K_{1} \cdot\left(k_{2}-k_{e l}\right)}{k_{2}+k_{3}-k_{e l}}
$$

The values of $V 1, V 2$, and $k_{2}^{*}+k_{3}^{*}$ can be estimated by nonlinear least-squares methods [48] given the tissue radioactivities at the end of the experiments $\left(C_{I}^{*}(T)\right)$, the plasma radioactivity curves $\left(C_{p}^{*}(t)\right)$, and the elimination constant $k_{e l}$. In fact, the equations for $V_{1}$ and $V_{2}$ were inserted directly into equation (2.15) and the nonlinear least-squares program was used to estimate $K^{*}$ directly. In theory, even $k_{e l}$ could be estimated from the least-squares methods, but we elected to use the published value of $0.017 \mathrm{~min}^{-1}$ [31] for the following reason. Assuming that the value of $0.017 \mathrm{~min}^{-1}$ is approximately correct, the half-life of the 5-HIAA pool in the brain (which is relatively constant for different brain structures [31]) will be roughly equal to $\ln 2 / 0.017=40.77$ minutes. My experiments were of a maximum of 60 minutes duration, which is only 1.5 half-lives of the 5-HIAA pool. In order to get a reliable estimate of $k_{e l}$, longer experiments would have to be performed (preferably up to several half-lives) in order to accurately follow the loss of the tracer from the brain. Since this was not done, we elected to use the published value for $k_{e l}$.

\subsection{Determination of the Lumped Constant}

The actual determinations of $K^{*}$ in various brain regions for rats given $\alpha$ - $\left[{ }^{14} C\right]$-methylL-tryptophan as the tracer were made previously [19] [34]. In order to determine the lumped constant, the method I used was a combination of the methods described in the previous sections. Radioactive tryptophan rather than $\alpha$-methyl-tryptophan was used as a tracer to determine the rate of 5-HT synthesis directly (since the endogenous substrate is being used, the lumped constant in this case is equal to 1 by definition). The rate of 5 -HT synthesis was then used to estimate the lumped constant 
for the $\alpha$-methyl-tryptophan method. $K^{*}$ was calculated by the method of the last section using $k_{e l}$ (subject to a correction for tryptophan incorporation into protein, as described below). This method works equally well for experiments with and without probenecid, since $k_{e l}$ is accounted for; in the case of probenecid-treated rats $k_{e l}$ was set to zero since efflux of 5-HIAA from the brain to the CSF was blocked by the drug. Note however that for probenecid-treated rats, the linearized method using time-activity plots could also have been used. Given this $K^{*}$, the rate of serotonin synthesis is given by:

$$
R=C_{p} \cdot K^{*}=K^{C_{p}}
$$

If we assume that in both groups of rats, under similar treatments, the steady-state rate of serotonin synthesis is the same, we can calculate the lumped constant as follows:

$$
L C=\frac{K^{C_{p}}\left(\alpha-\cdot m_{i} e t h y l-5 H T\right)}{K^{C_{p}(5 H T)}}
$$

This method is applied separately for each brain region, allowing us to test the hypothesis that the lumped constant is in fact constant over different brain regions, as one would expect.

I performed a second series of experiments in which an intraperitoneal injection of the drug probenecid was given prior to tracer injection. Probenecid blocks 5-HIAA efflux into plasma [49], thus blocking tracer efflux from the tissue by any route other than the reversible transfer of tryptophan from the extracellular compartment to the plasma (which is accounted for in the model). One would expect that probenecid treatment would not alter the lumped constant, which should only reflect the differences in the kinetic properties of the rate-limiting enzyme with respect to the tracer and the tracee [21]. However, since probenecid treatment in our protocol can affect 
other physiological parameters (such as $\mathrm{pO}_{2}$ and plasma tryptophan concentration) known to affect tryptophan synthesis, differences in the calculated lumped constants do not necessarily reflect true changes in the lumped constant, for the following reasons. The calculations of the lumped constant using probenecid used $K^{C p}$ values for $\alpha-\left[{ }^{14} C\right]$ methyl-5-HT which were obtained using rats not treated with probenecid; this is reasonable if the only relevant effect of probenecid is to block 5-HIAA efflux but may introduce errors if other relevant physiological parameters are different between the two groups (untreated rats with $\alpha$ - $\left[{ }^{14} C\right]$ methyl-L-tryptophan as a tracer and probenecid-treated rats with L- $\left[{ }^{14} C\right]$-tryptophan as a tracer) are significantly different (see Results and Discussion).

\subsubsection{Determination of the Regional Rates of Tryptophan Incorporation into Proteins}

One difficulty with the method that must be addressed is that tryptophan is not only metabolized into serotonin, but is also incorporated into proteins (unlike $\alpha$ methyl-tryptophan, which is not incorporated into proteins to any significant extent [19]). In other words, the compartmental model, instead of having one "irreversible" compartment, actually has two. Note that in this case we are ignoring the very small transfer of radioactivity from the protein compartment back to the precursor pool(s) of tryptophan and thus consider tracer incorporation into protein to be irreversible over the duration of the experiments. In order to subtract out this contribution, I prepared replicate brain slices for all rats used and washed half of them in $20 \%$ TCA, which precipitates proteins but washes out non-protein-bound radioactivity (see Materials and Methods). These washed brain slices, as well as unwashed brain slices, were exposed to $\mathrm{x}$-ray film to determine both total tissue radioactivity and proteinbound tissue radioactivity. Protein-bound radioactivity can be subtracted from total 
radioactivity for each brain region to yield non-protein-bound radioactivity, which is the $C_{I}^{*}(T)$ used to calculate $K^{*}$ for serotonin as described above. In addition, we can use the linearized time-activity method described above to calculate the local rates of L-tryptophan incorporation into brain proteins. In this case we use protein-bound radioactivity as $C_{I}^{*}(T)$, with $\Theta(T), C_{p}$, and $C_{p}^{*}$ the same as above, and we obtain the regional $K^{*}$ from equation (2.9) which can then be multiplied by the average $C_{p}$ to get the regional $K^{C_{p}}$ which is equal to the regional rate of L-tryptophan incorporation into proteins. These values are a "bonus" of the procedure used to get the lumped constant for the $\alpha-\left[{ }^{14} C\right]$ methyl-L-tryptophan method. Also, since TCA-washing of slices removes the precursor pool from the brain slices (since it is not bound to protein) equation (2.9) was fit using a $V_{a p p}$ of zero (the apparent volume of distribution of the precursor pool is zero); i.e. the regression line intersects the origin.

\subsection{Relationship of Rate Constants to Actual En- zyme Activities}

It must be pointed out that the three-compartment model is an operational model, which is consistent with what is known about the metabolic steps involved in serotonin synthesis. The method does not, however, claim that the rate constants represent actual kinetic parameters such as the $K_{M}$ or $V_{\max }$ values of the rate-limiting enzymes or some combination of them. It is to be expected that these parameters will be the major determinants of the rate constants of the model, but since the real system might have many physical compartments other than the ones modelled whose rate constants are unavailable, the model's rate constants are essentially a gross approximation to the large-scale kinetic behavior of the system, and can only be justified by the extent to which the model empirically fits the data. This is even more true for the method 
involving time-activity plots, which directly calculates $K^{*}$, itself a function of several other rate constants.

\subsection{Other Ways to Determine the Lumped Con- stant}

\subsubsection{Sokoloff's method}

The idea of a "lumped constant" was first presented by Sokoloff et. al. for the 2-deoxyglucose method in [21]. In that paper it was demonstrated that if $\left[{ }^{14} \mathrm{C}\right]$ deoxyglucose is administered to the animal to maintain $C_{p}^{*}$ at a constant level for a sufficiently long time, the lumped constant could be calculated by:

$$
L C=\left(\frac{E^{*}}{E}\right)\left(\frac{C_{A}^{*}}{C_{A}}\right) /\left(\frac{C_{p}^{*}}{C_{p}}\right)
$$

where $E=\frac{C_{A}-C_{V}}{C_{A}}$ and $E^{*}=\frac{C_{A}^{*}-C_{V}^{*}}{C_{A}^{*}}$ are the cerebral extraction ratios for glucose and $\left[{ }^{14} \mathrm{C}\right]$-deoxyglucose, respectively.

In order to use this method, an appropriate infusion schedule must be designed which will make $C_{p}^{*}$ reach a constant value and stay there for long enough for the above equation to be valid ( $>30$ minutes in Sokoloff's paper). In addition, both arterial blood samples and venous blood samples (from the femoral artery and the confluence of the sinuses, respectively) must be taken. One difficulty is obtaining the venous blood samples, which is surgically somewhat tricky. In addition, the extraction ratios for L-tryptophan are small, as mentioned above leading to large errors since the extraction ratio for unlabelled tryptophan is in the denominator of the above expression. The method described previously does not require difficult surgical techniques, is not dependent on the extraction ratios, and also provides extra 
information about regional rates of tryptophan incorporation into protein.

\subsubsection{Method of Phelps et. al.}

In the paper of Phelps et. al. [46] a different method was used to estimate the lumped constant in conjunction with PET studies on human volunteers. In their method, the LC was initially arbitrarily set to 1 and values for local cerebral metabolic rates of glucose (LCMRGlc) were calculated. These were then integrated across the brain to give a whole-brain CMRGlc. The ratio of this value to the average CMRGlc calculated by other methods is the lumped constant. This method has some similarity to our method, in that the metabolic rate of the tracer and the tracee were calculated separately. However, our method calculates the LC separately for all brain regions investigated, allowing us to test whether the $\mathrm{LC}$ is constant in different brain regions, which is not possible if whole-brain metabolism of the tracee is used to get the LC. 


\section{Chapter 3}

\section{Materials and Methods}

\subsection{Radiopharmaceuticals}

${ }^{14} \mathrm{C}$-L-tryptophan, labeled on the indole ring, was obtained from Amersham Corp. (Arlington Heights, IL, U.S.A.).

\subsection{Surgical Procedures}

33 Female Wistar rats weighing between 190-250 $g$ were used. Animals were fasted the night before the experiment. Under light (1.5\%) halothane anaesthesia, the femoral artery and vein were catheterized with PE-50 polyethylene catheters. The abdomen and hind legs of the rat were covered with a plaster cast and the rat was allowed to wake up. All rats were awake at least 2 hours before being injected with tracer. Physiological values (arterial $p \mathrm{H}, \mathrm{P}_{a} \mathrm{CO}_{2}, \mathrm{P}_{a} \mathrm{O}_{2}$, blood pressure, hematocrit and rectal temperature were measured before the beginning of the experiment, and hematocrits, $p H, \mathrm{P}_{a} \mathrm{CO}_{2}$, and $\mathrm{P}_{a} \mathrm{O}_{2}$ were measured at the end of the experiments, except for experiments of 2 or 5 minutes duration, in which hematocrits and blood gases were only taken at the beginning of the experiment. $2 \mathrm{ml}$ of tracer was injected into 
the femoral vein of the animal in a 2 minute bolus, except for the experiments of 5 minute duration, in which the tracer was injected as a 5 minute bolus. At the end of each experiment, the animals were killed by decapitation, and the brain was rapidly removed and frozen in liquid freon. Brain slices $(30 \mu \mathrm{m})$ were prepared in a cryostat and exposed for three weeks on x-ray film along with standards to obtain autoradiograms, which were used to determine total tissue concentrations of tracers (see below). In addition, replicate brain slices were prepared for all regions and washed 3 times in $20 \%$ trichloroacetic acid to remove all non-protein-bound radioactivity. These slices were air dried overnight, covered with mylar to prevent residual traces of TCA from darkening the film, and exposed along with mylar-covered standards for three weeks on $\mathrm{x}$-ray film to obtain autoradiograms. These autoradiograms yielded tissue concentrations of non-protein-bound radioactivity.

Aliquots of plasma samples were put into scintillation fluid and plasma radioactivity was determined by liquid scintillation counting. Plasma samples were also used to determine plasma tryptophan by HPLC (see below). In addition, plasma samples were also filtered to separate free tryptophan from protein-bound tryptophan, and these samples were also analyzed by HPLC. This procedure enabled us to calculate the percent free tryptophan in plasma.

\subsection{Probenecid treatment}

For 16 out of the 33 rats, $200 \mathrm{mg} / \mathrm{kg}$ probenecid (Sigma Chemical Company, St. Louis) was injected intraperitoneally after surgery at least 1 hour before the experiment. 


\subsection{Plasma Tryptophan Determination}

Plasma amino acid concentrations were determined using a Hewlett-Packard series 1050 HPLC apparatus.

\subsection{Data Analysis}

The autoradiographic images were digitized using a microcomputer-based image analysis system (The Image Calculator, Soquelec Ltd., Montreal) which consisted of a video camera, a frame grabber, an IBM AT compatible computer and the appropriate software. Optical densities were converted into tissue tracer concentrations (nCi/gram) using a calibration curve relating the optical densities of ${ }^{14} \mathrm{C}$ standards with measured ${ }^{14} C$ concentrations in rat brain.

The plasma radioactivity curve was used to calculate $\Theta$, the time integral of plasma radioactivity using a nonlinear curve fitting program employing a Stineman spline interpolation routine [50]. Pooled results from all experiments were combined into time-activity plots for each brain region (described in the Theory section). The $\mathrm{x}$-axis of these plots was equal to $\Theta$, while the $\mathrm{y}$-axis was equal to $\frac{C_{I}^{*}(T)}{C_{p}^{*}(T) \cdot f_{f}^{*}} . C_{I}^{*}(T)$ is the tissue radioactivity, $C_{p}^{*}(T)$ is the total plasma radioactivity at the end of the experiment, and $f_{f}$ is the ratio of free tryptophan to total tryptophan in the plasma, calculated separately for each experiment. For determination of $K^{*}$ values for 5 -HT synthesis the method involving $k_{e l}$ was used as described in the theory section; the parameters of this method were calculated using standard nonlinear least-squares methods [48] and the standard deviations of the estimated parameter values were calculated as the square roots of the diagonal elements in the variance-covariance matrix. For determination of $K^{*}$ values for the rate of tryptophan incorporation into protein, linear regressions constrained to go through the origin were used and the slope 
and standard error of the slope were calculated by standard methods [51]. $K^{*}$ values were used to calculate $K^{C p}$ values as described in the Theory section. The $K^{C p}$ values for tryptophan incorporation into protein give the rate of tryptophan incorporation into protein directly. The $K^{C p}$ values for 5-HT synthesis were used along with $K^{C p}$ values for $\alpha-\left[{ }^{14} C\right]$-methyl-L-tryptophan (calculated previously $[19,20,34]$ ) to get regional estimates of the lumped constant as described in the theory section. These were then used to generate estimates of the global lumped constant for untreated and probenecid-treated rats, as discussed in the Results section.

The apparent $K_{M}$ of the LNAA transporter for tryptophan was calculated using the following equation [26]:

$$
K_{M}^{a p p}=K_{M}^{t r p}\left(1+\sum \frac{[A A]}{K_{M}^{A A}}\right)
$$

using the individual absolute $K_{M}$ 's determined in [25]. PCR, which stands for "plasma competitor ratio", is calculated as:

$$
P C R=\frac{V_{\max } \cdot[T r p]}{K_{M}^{a p p}+[T r p]}
$$

In this case, the $V_{\max }$ values were obtained from [26]. PCR ( $\left.\mathrm{nmol} / \mathrm{g} / \mathrm{min}\right)$ represents the rate of carrier facilitated influx of an amino acid which competes with other amino acids in a given transporter. A higher relative value of PCR for a given amino acid means that the carrier has a higher preference for that amino acid and thus the rate of transport is greater. 


\section{Chapter 4}

\section{Results}

\subsection{Overview}

As described in the previous sections, in order to calculate the lumped constant for the $\alpha$-methyl-tryptophan method for a given brain region, the following information must be known:

1. The net unidirectional influx constant $\left(K_{A M T r p}^{*}\right)$ for the net unidirectional transfer of $\alpha$-methyl-tryptophan into an irreversible compartment for that brain region, calculated as described in the Theory section.

2. The average plasma tryptophan concentration $\left(C_{p}\right)$ for the series of experiments using $\alpha-\left[{ }^{14} \mathrm{C}\right]$ methyl-L-tryptophan, which when multiplied by $K^{*}$ gives $K^{C p}$ for $\alpha$-methyl-tryptophan, which however is not the true rate of either 5 -HT synthesis or $\alpha$-methyl-5HT synthesis since in this case we are multiplying the $K^{*}$ for $\alpha$-methyl-5-HT by the plasma concentration of free tryptophan. This value will differ from the true rate of 5 -HT synthesis by a factor equal to the lumped constant. 
3. The net unidirectional influx constant $\left(K_{T r p}^{*}\right)$ for the synthesis of 5 -HT for that brain region, calculated from time-activity plots as described in the Theory section.

4. The average plasma tryptophan concentration $\left(C_{p}\right)$ for the series of experiments using L- $\left[{ }^{14} \mathrm{C}\right]$ tryptophan, which when multiplied by $K_{T r p}^{*}$ gives $K^{C p}$ for 5 -HT.

With this information, the LC is simply the ratio of $K^{C p}$ 's for $\alpha$-methyl-5-HT and 5 -HT as described in the Theory section.

The regional $K^{C p}$ 's for $\alpha^{-}\left[{ }^{14} C\right]$ methyl-5-HT have been calculated previously [20, 34]. I include the relevant results below, since they are needed to calculate the lumped constant. The lumped constant is calculated twice, once using data obtained from rats not treated with probenecid, and once using data obtained from rats treated with probenecid to block tracer efflux from the brain. 


\subsection{Results from previous work}

Table 1 consists of results from tracer experiments using $\alpha-\left[{ }^{14} C\right]$ methyl-L-tryptophan as tracer (described in $[19,20]$ ). All subsequent data consists of results from tracer experiments using $\mathrm{L}-\left[{ }^{14} C\right]$-tryptophan as tracer.

Table 1. Rates of uptake of $\left.\alpha-{ }^{4} C\right]$ methyl-L-tryptophan $\left(K^{C p}\right)[20,34]$; all values \pm S.D., $n=28$

\begin{tabular}{|l|c|}
\hline Structure & $K^{C p}(\mathrm{pmol} / \mathrm{g} / \mathrm{min})$ \\
\hline raphe obscurus & $30.63 \pm 3.46$ \\
inferior olive & $13.71 \pm 3.22$ \\
raphe pallidus & $22.90 \pm 3.50$ \\
raphe magnus & $19.67 \pm 4.77$ \\
locus coeruleus & $18.95 \pm 3.58$ \\
inferior colliculus & $11.20 \pm 2.50$ \\
visual cortex & $12.16 \pm 1.79$ \\
pontine raphe & $32.90 \pm 3.93$ \\
dorsal raphe (VM) & $89.76 \pm 8.82$ \\
dorsal raphe (4 parts) & $55.90 \pm 6.44$ \\
medial raphe & $62.34 \pm 7.51$ \\
superior colliculus & $14.54 \pm 3.22$ \\
ventral tegmental area & $19.55 \pm 2.50$ \\
medial geniculate nucleus & $18.00 \pm 2.62$ \\
substantia nigra & $16.81 \pm 2.86$ \\
dorsal hippocampus & $21.22 \pm 2.98$ \\
ventral hippocampus & $19.67 \pm 2.26$ \\
hippocampus - CA3 & $22.05 \pm 4.41$ \\
\hline
\end{tabular}


Table 1 (continued). Rates of uptake of $\alpha-\left[{ }^{14} C\right]$ methyl-L-tryptophan $\left(K^{C p}\right)$ [20, 34]; all values \pm S.D., $n=28$

\begin{tabular}{|l|c|}
\hline Structure & $K^{C p}(\mathrm{pmol} / \mathrm{g} / \mathrm{min})$ \\
\hline auditory cortex & $16.45 \pm 2.26$ \\
medial forebrain bundle & $17.52 \pm 3.70$ \\
ventral thalamus & $16.69 \pm 2.62$ \\
hypothalamus & $15.97 \pm 3.10$ \\
parietal cortex & $17.76 \pm 3.10$ \\
sensory-motor cortex & $13.47 \pm 2.15$ \\
caudate - medial & $12.16 \pm 1.55$ \\
caudate - lateral & $20.03 \pm 3.34$ \\
nucleus accumbens & $17.76 \pm 2.15$ \\
olfactory cortex & $26.94 \pm 4.77$ \\
\hline
\end{tabular}




\subsection{Physiological parameters}

The average values for physiological parameters for the rats used in my experiments are shown in tables 2 and 3 . As can be seen, probenecid treatment caused a significant alteration in both blood pressure and blood gases. The alterations in blood $\mathrm{pO}_{2}$ could potentially have had an effect on 5-HT synthesis, since oxygen is a substrate for the enzyme tryptophan-5-hydroxylase (see Introduction). There was also a significant decrease in blood $\mathrm{CO}_{2}$; however, this should not have had any direct effect on the rate of 5-HT synthesis, since $\mathrm{CO}_{2}$ is not directly involved in the metabolic pathway of 5-HT synthesis. In table 4 the amino acid concentrations of both probenecidtreated and control rats are shown. Clearly probenecid treatment caused a number of changes in the concentrations of amino acids known to use the large neutral amino acid (LNAA) transporter as well as very large changes in the concentration of free tryptophan.

In addition, the apparent $K_{M}$ of the LNAA transporter for tryptophan decreased significantly (table 4), indicating that the affinity of the LNAA transporter for tryptophan would have been proportionally higher during probenecid treatment as a consequence of the higher free tryptophan levels in the plasma. The PCR for free trypto-

phan (PCR(free)) also increased significantly (table 4), indicating a greater preference of the transporter for free tryptophan. The change in free tryptophan levels proved to cause a large change in the regional lumped constant values. This may be due to the fact that the rats used in the experiments using $\alpha$ - $\left[{ }^{14} C\right]$ methyl-L-tryptophan as the tracer were not treated with probenecid, so a direct comparison is not really appropriate. Nevertheless, when the rate of 5-HT synthesis in probenecid-treated rats was normalized for the difference in free tryptophan levels, as shown later in this chapter, the lumped constant values were not significantly different. 
Table 2. Average values of physiological parameters for rats not treated with probenecid; all values \pm S.D.

\begin{tabular}{|l|c|c|}
\hline Parameter & Average value & n \\
\hline Weight $(g)$ & $203.4 \pm 16.4$ & 11 \\
Hematocrit & $0.43 \pm 0.02$ & 17 \\
Blood Pressure $(\mathrm{mmHg})$ & $127.5 \pm 12.2$ & 17 \\
Rectal temperature $\left({ }^{\circ} \mathrm{C}\right)$ & $36.5 \pm 0.6$ & 17 \\
Arterial $p H$ & $7.46 \pm 0.03$ & 17 \\
Arterial $\mathrm{P}_{\mathrm{O}_{2}}(\mathrm{mmHg})$ & $93.33 \pm 4.99$ & 17 \\
Arterial $\mathrm{P}_{\mathrm{CO}_{2}}(\mathrm{mmHg})$ & $34.71 \pm 2.37$ & 17 \\
\hline
\end{tabular}

Table 3. Average values of physiological parameters for rats treated with probenecid; all values $\pm S . D$.; $p$ values calculated from 2-tailed $t$-test; $N S=$ not significant

\begin{tabular}{|l|c|c|c|}
\hline Parameter & Average value & $\mathrm{n}$ & p (+pro vs -pro) \\
\hline Weight $(g)$ & $199.2 \pm 9.2$ & 16 & $\mathrm{NS}$ \\
Hematocrit & $0.42 \pm 0.03$ & 16 & $\mathrm{NS}$ \\
Blood Pressure $(\mathrm{mmHg})$ & $111.6 \pm 12.7$ & 15 & $<0.05$ \\
Rectal temperature $\left({ }^{\circ} \mathrm{C}\right)$ & $36.0 \pm 0.8$ & 16 & $\mathrm{NS}$ \\
Arterial $p H(m m H g)$ & $7.48 \pm 0.04$ & 16 & $<0.01$ \\
Arterial $\mathrm{PO}_{2}(\mathrm{mmHg})$ & $115.89 \pm 9.60$ & 15 & $<0.01$ \\
Arterial $\mathrm{P}_{\mathrm{CO}_{2}(\mathrm{mmHg})}(\mathrm{30.57} \pm 2.58$ & 16 & $<0.05$ \\
\hline
\end{tabular}


Table 4. Plasma Amino Acid analysis; all values \pm S.D.; NS = not significant; p values calculated by $A N O V A$

\begin{tabular}{|l|c|c|c|}
\hline Amino Acid & $\begin{array}{c}\text { Conc. (nmol/ml) } \\
\text { (no probenecid) }\end{array}$ & $\begin{array}{c}\text { Conc. (nmol/ml) } \\
\text { (with probenecid) }\end{array}$ & p \\
\hline Valine & $267 \pm 93$ & $182 \pm 35$ & $<0.002$ \\
Isoleucine & $132 \pm 44$ & $79 \pm 20$ & $<0.0002$ \\
Leucine & $214 \pm 73$ & $139 \pm 34$ & $<0.0008$ \\
Phenylalanine & $82 \pm 29$ & $75 \pm 7$ & NS \\
Tyrosine & $60 \pm 22$ & $53 \pm 6.5$ & NS \\
Methionine & $46 \pm 17$ & $48 \pm 15$ & NS \\
Tryptophan & $80 \pm 19$ & $51 \pm 13$ & $<0.00002$ \\
Tryptophan $($ Free $)$ & $7.56 \pm 2.8$ & $12.02 \pm 2.48$ & $<0.0004$ \\
\hline$K_{M}($ apparent $)(\mu \mathrm{M})$ & $507 \pm 153$ & $404 \pm 46$ & $<0.015$ \\
PCR (nmol $/ \min / g)$ & $0.292 \pm 0.168$ & $0.524 \pm 0.112$ & $<0.0006$ \\
\hline PCR (free) * $(\mathrm{nmol} / \mathrm{min} / \mathrm{g})$ & & $2.00 \pm 0.29$ & $<0.003$ \\
\hline
\end{tabular}

* calculated using free tryptophan concentration. 


\subsection{Results for serotonin synthesis}

\subsubsection{Sample time-activity plots}

Figure 4.1 shows representative autoradiograms of rat brain slices obtained from an experiment in which the rat was given a 2 minute bolus injection of $\left[{ }^{14} C\right]$-tryptophan and was sacrificed 60 minutes after the beginning of the injection. The slice on the right was washed three times in $20 \%$ TCA to remove all non-protein-bound radioactivity, as described in the Materials and Methods section. The slice on the left was not washed, so the total radioactivity present in brain tissue can be calculated from the autoradiogram.

In this section I include sample time-activity plots for three brain regions for both the control and probenecid-treated group of rats. The $\mathrm{x}$-axis is the "exposure time" $\Theta(T)$, which is the integral of the plasma radioactivity curve divided by the plasma radioactivity value at the kill time (see Theory section). The y-axis is the apparent volume of distribution of the tracer, which equals the ratio of the tissue radioactivity to the plasma radioactivity multiplied by the fraction of free tryptophan $\left(\frac{C_{f}^{*}(T)}{C_{p}^{*}(T) \cdot f_{f}}\right)$ at kill time. The slope of the graph is initially steep, representing the large initial rate of tracer uptake into the brain (since the equilibrium between the concentrations of tracer in the plasma compartment and the extracellular compartment is not yet complete).

After about 20 minutes (exposure time), the uptake of tracer reached an apparent steady state, and the slope of the time-activity curve is the value of $K^{*}$ for the probenecid-treated rats (it is slightly less than $K^{*}$ for the untreated rats due to efflux of the tracer to the CSF at longer experimental times). The regression curve was calculated using a nonlinear regression algorithm to fit the parameters of the full model incorporating $k_{e l}$ as discussed in the Materials and Methods and Theory 
sections. $K^{*}$ values were obtained from these procedures as described in the Theory section and used to calculate the $K_{C p}$ values tabulated below.

It should be noted that the fitted curves in the following figures were obtained by a numerical integration procedure which can result in a relatively unsmooth appearance of the curves (figs. 4.2 to 4.7 ). 

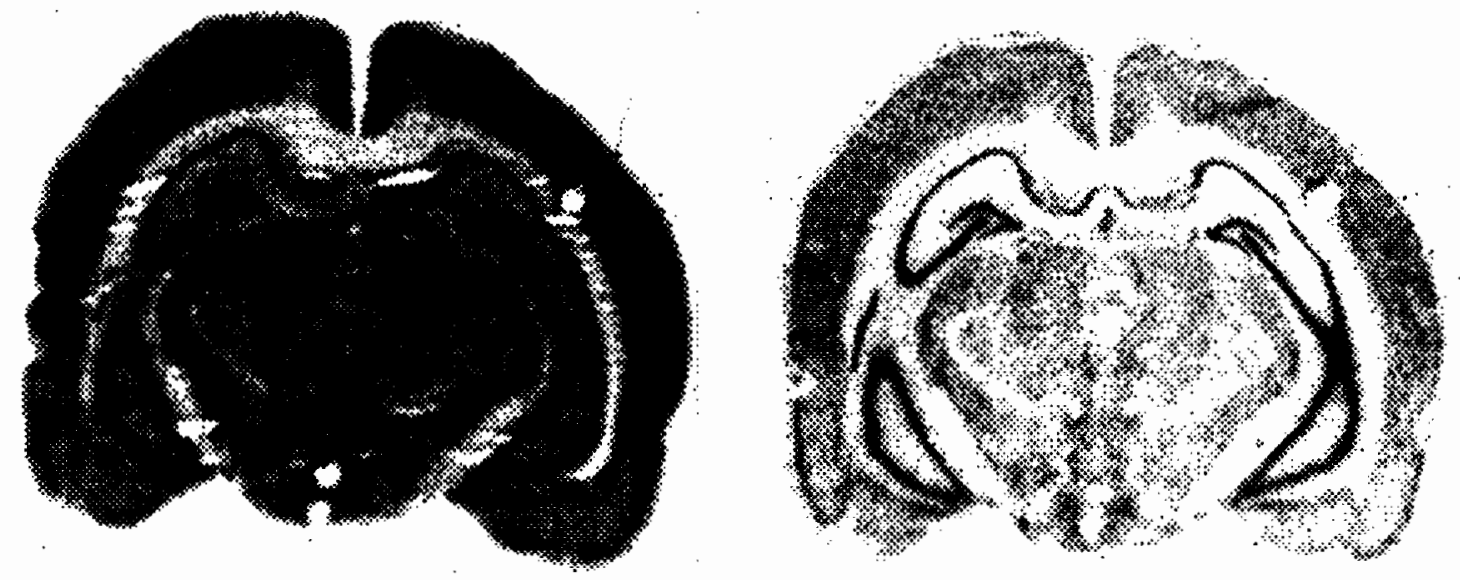

Figure 4.1: Representative autoradiograms of rat brain $60 \mathrm{~min}$. after treatment with (14C)-tryptophan. Left: unwashed brain. Right: brain slice washed in $20 \%$ TCA. 


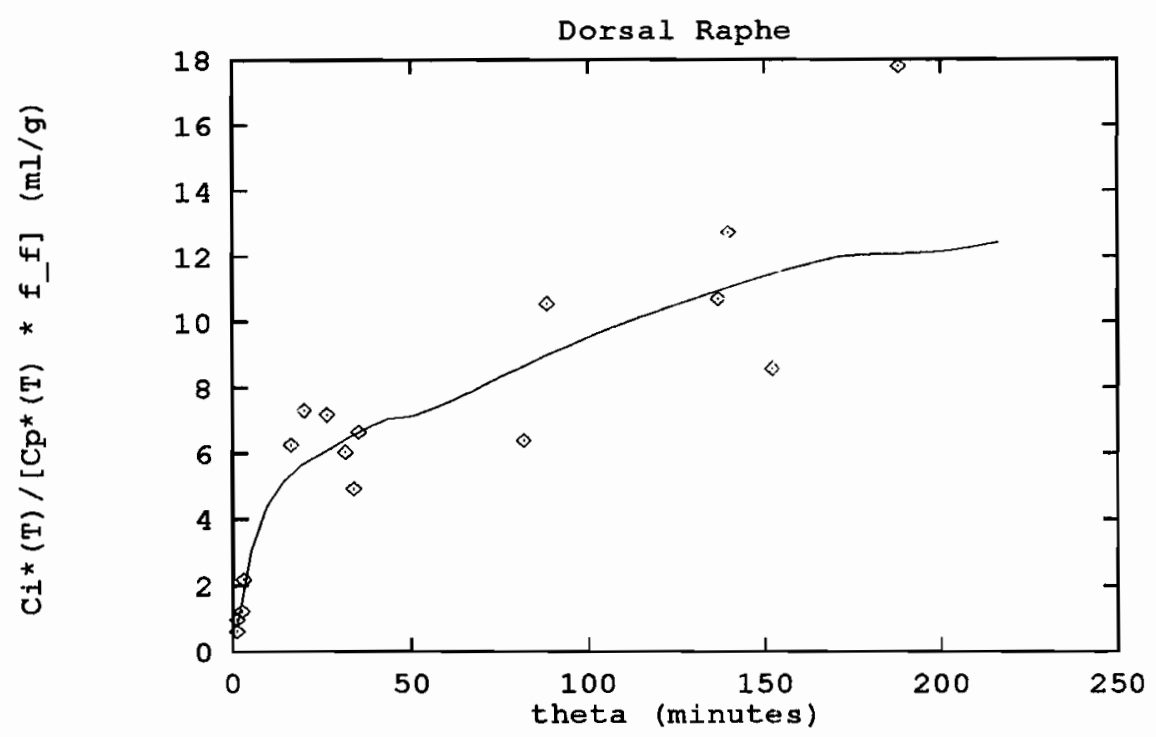

Figure 4.2: Time-activity plot for serotonin synthesis for rats not treated with probenecid (dorsal raphe nucleus).

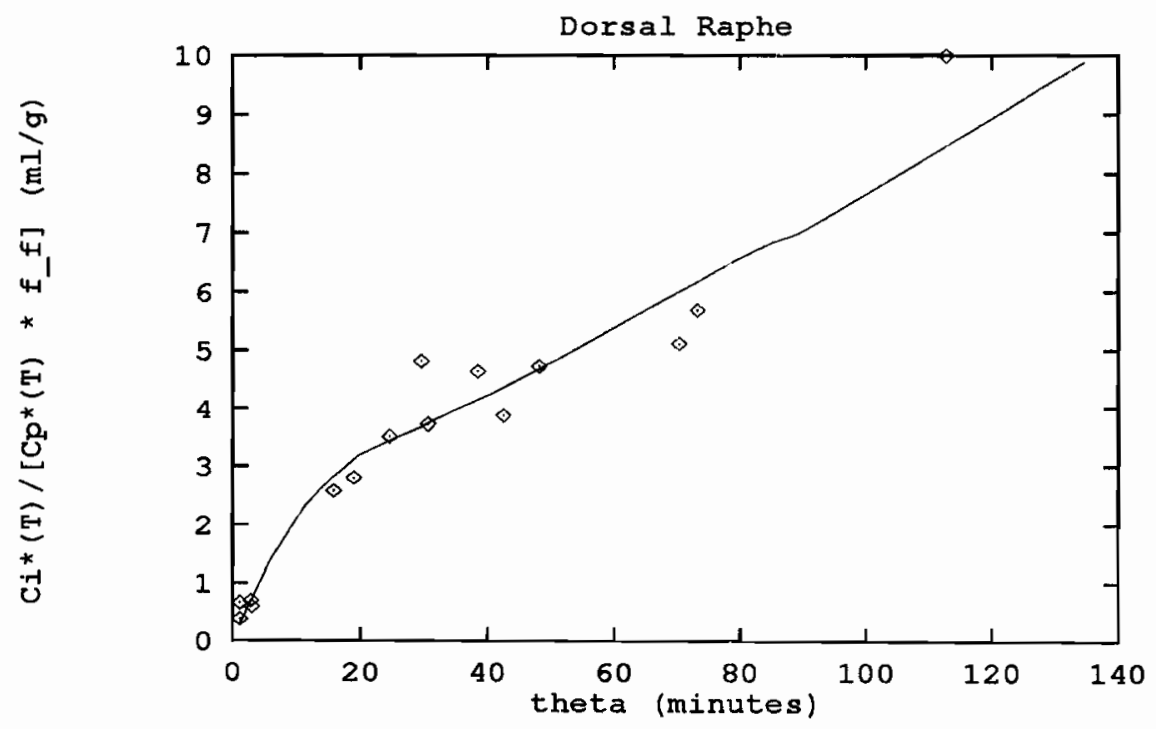

Figure 4.3: Time-activity plot for serotonin synthesis for rats treated with probenecid (dorsal raphe nucleus). 


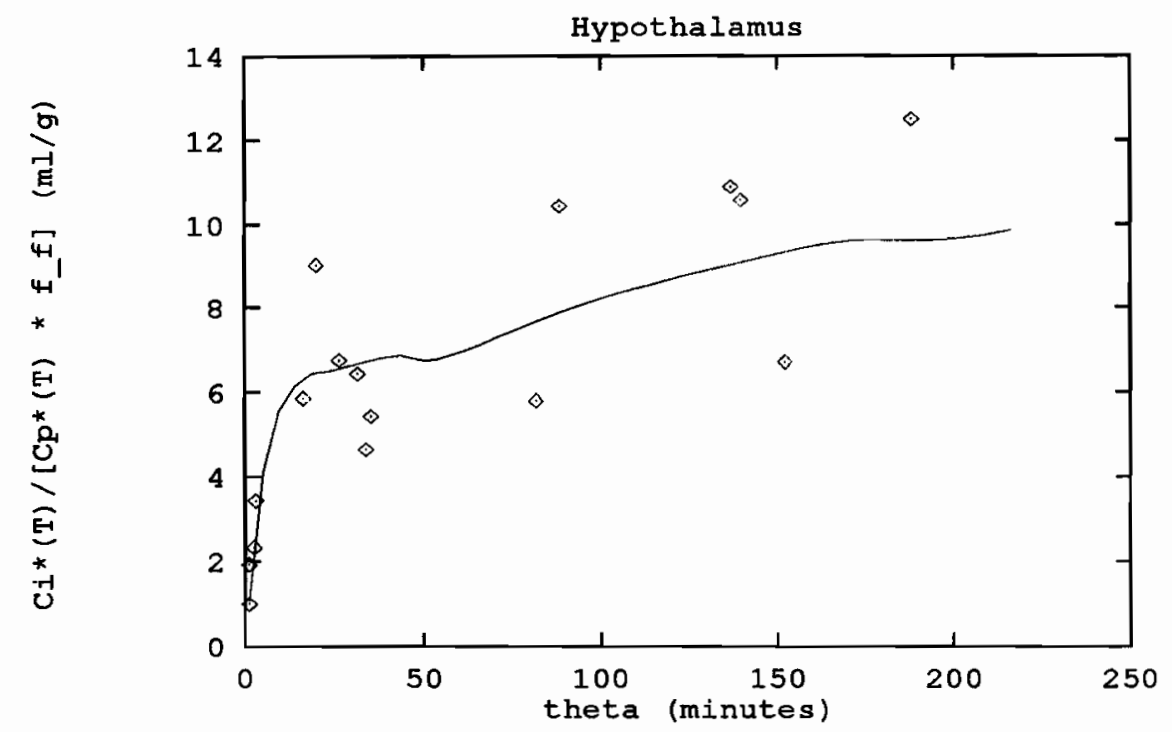

Figure 4.4: Time-activity plot for serotonin synthesis for rats not treated with probenecid (hypothalamus).

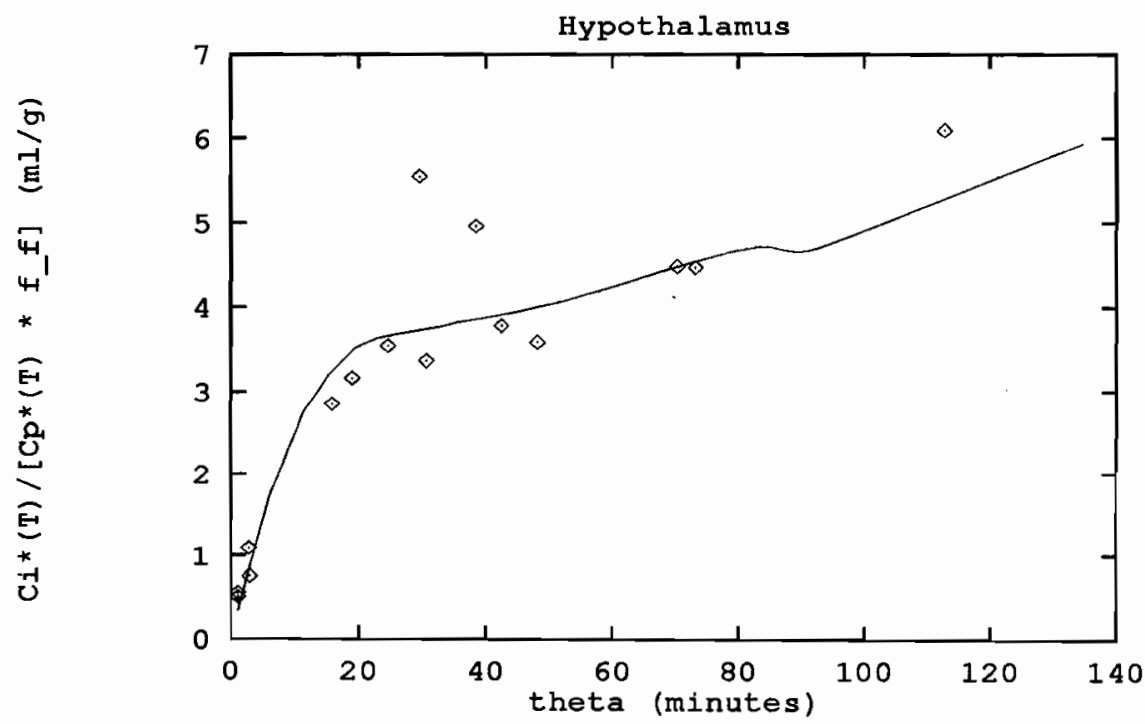

Figure 4.5: Time-activity plot for serotonin synthesis for rats treated with probenecid (hypothalamus). 


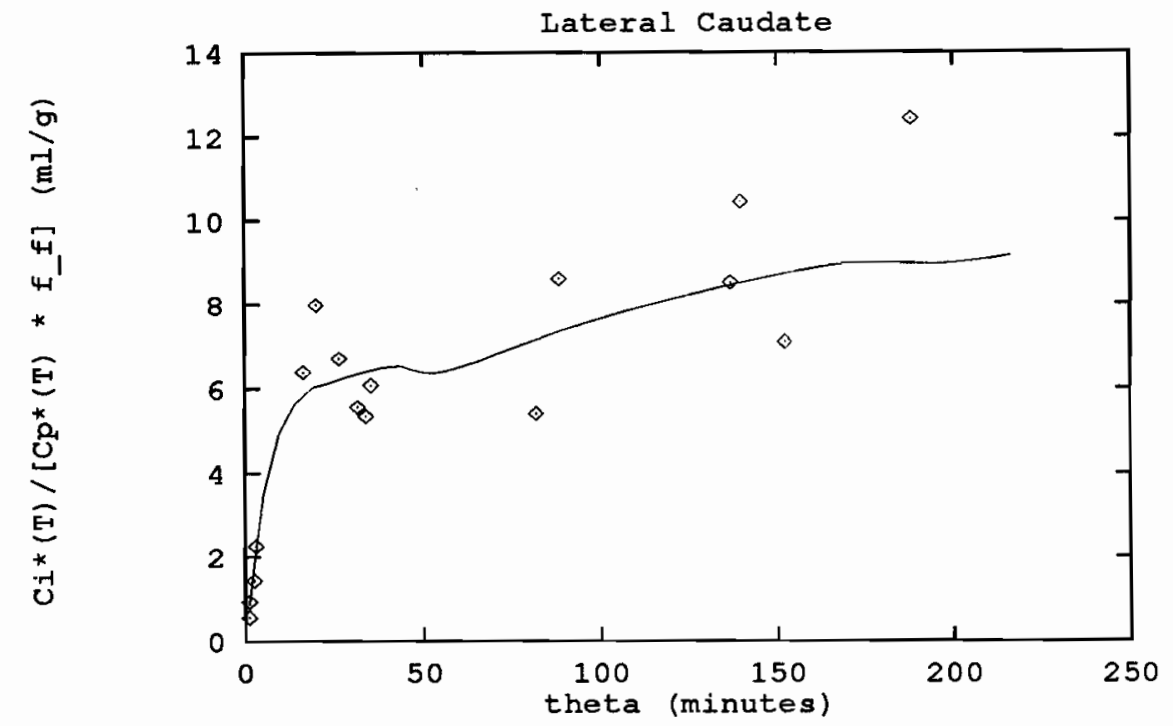

Figure 4.6: Time-activity plot for serotonin synthesis for rats not treated with probenecid (lateral caudate nucleus).

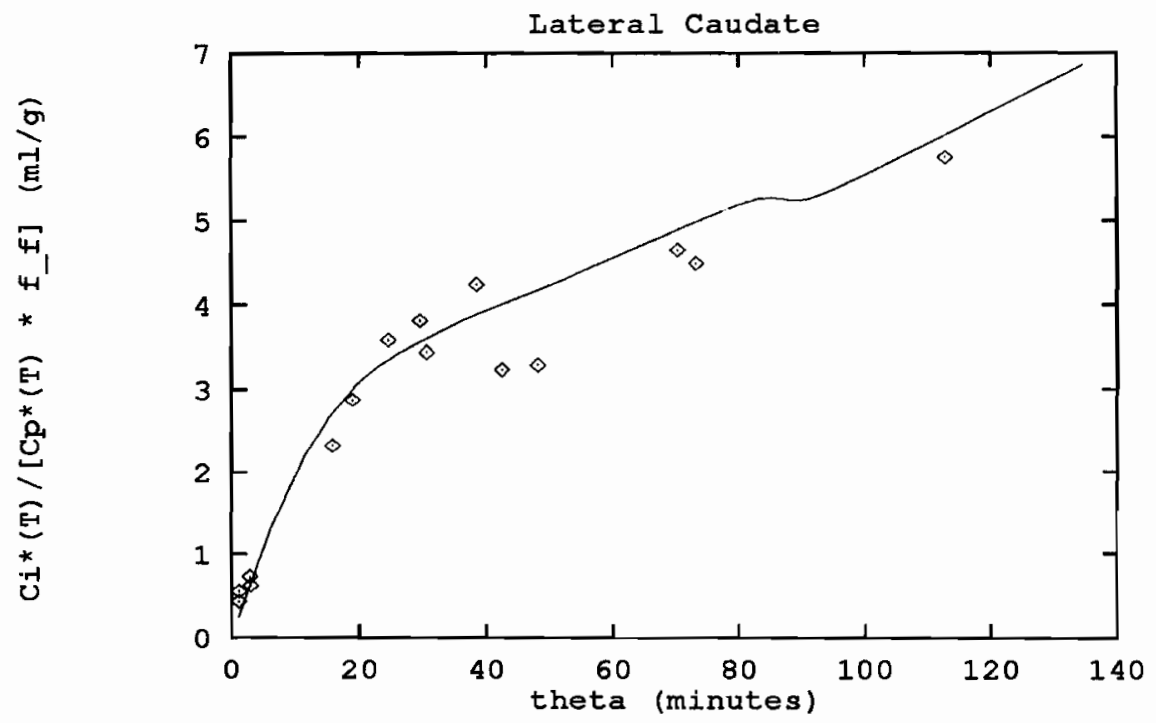

Figure 4.7: Time-activity plot for serotonin synthesis for rats treated with probenecid (lateral caudate nucleus). 


\subsubsection{Rates of Serotonin Synthesis and Regional Lumped Constants}

In tables 5 and 6 , I have included the rates of 5 -HT synthesis for control and probenecid-treated rats, which is equal to $K^{C p}$ when $\mathrm{L}-\left[{ }^{14} C\right]$-tryptophan is used as a tracer. The lumped constant $(\mathrm{LC})$ is equal to the ratios of the $K^{C p}$ 's for $\alpha-\left[{ }^{14} C\right]$ methyl-L-tryptophan-treated rats (tabulated above in table 1 ) and L- $\left[{ }^{14} C\right]$ tryptophan-treated rats. It can be seen that there is a considerable degree of variation in the regional $K^{C p}$ 's with both $\alpha$ - $\left[{ }^{14} C\right]$ methyl-L-tryptophan (table 1 ) and ${ }^{14} C$ L-tryptophan used as the tracer, leading in this case to a high variability in the lumped constant. The regional LC's are equal to the ratios of the $K^{C p}$ 's for $\alpha$ $\left[{ }^{14} C\right]$ methyl-L-tryptophan-treated rats and the $K^{C p}$ 's for $\mathrm{L}-\left[{ }^{14} C\right]$-tryptophan-treated rats as described above. 
Table 5. Rates of incorporation of $L-\left[{ }^{4} C\right]$-tryptophan into serotonin $\left(K^{C_{p}}\right)$ for rats not treated with probenecid and regional lumped constants; all values \pm S.D., $n$ $=16$ data points in regression

\begin{tabular}{|l|c|c|}
\hline Structure & $K^{C p}(p m o l / g / m i n)$ & Regional LC \\
\hline raphe obscurus & $57.44 \pm 3.46$ & 0.53 \\
inferior olive & $61.09 \pm 4.90$ & 0.22 \\
raphe pallidus & $41.15 \pm 2.78$ & 0.56 \\
raphe magnus & $51.58 \pm 3.13$ & 0.38 \\
locus coeruleus & $38.53 \pm 4.04$ & 0.49 \\
inferior colliculus & $61.71 \pm 5.13$ & 0.18 \\
visual cortex & $65.82 \pm 5.35$ & 0.18 \\
pontine raphe & $66.84 \pm 3.60$ & 0.49 \\
dorsal raphe (ventromedial) & $76.77 \pm 4.91$ & 1.17 \\
dorsal raphe (all) & $74.75 \pm 4.54$ & 0.75 \\
medial raphe & $r 4.73 \pm 4.13$ & 0.83 \\
superior colliculus & $75.69 \pm 5.32$ & 0.19 \\
ventral tegmental area & $34.97 \pm 2.08$ & 0.56 \\
medial geniculate nucleus & $45.13 \pm 3.85$ & 0.40 \\
substantia nigra & $42.49 \pm 1.97$ & 0.40 \\
dorsal hippocampus & $29.41 \pm 2.62$ & 0.72 \\
ventral hippocampus & $46.90 \pm 3.07$ & 0.42 \\
hippocampus - CA3 & $44.08 \pm 3.45$ & 0.50 \\
\hline
\end{tabular}


Table 5 (continued). Rates of incorporation of $L-\left[{ }^{4} C\right]$ tryptophan into serotonin $\left(K^{C p}\right)$ for rats not treated with probenecid and regional lumped constants; all values $\pm S . D ., n=16$ data points in regression

\begin{tabular}{|l|c|c|}
\hline Structure & $K^{C_{p}}(\mathrm{pmol} / \mathrm{g} / \mathrm{min})$ & Regional LC \\
\hline auditory cortex & $43.52 \pm 3.26$ & 0.38 \\
lateral geniculate nucleus & $50.85 \pm 2.96$ & 0.34 \\
medial forebrain bundle & $51.12 \pm 2.54$ & 0.33 \\
ventral thalamus & $56.15 \pm 2.98$ & 0.28 \\
hypothalamus & $44.61 \pm 3.11$ & 0.40 \\
parietal cortex & $50.97 \pm 3.03$ & 0.26 \\
sensory-motor cortex & $40.71 \pm 2.89$ & 0.30 \\
caudate - medial & $38.29 \pm 2.94$ & 0.52 \\
caudate - lateral & $41.26 \pm 2.93$ & 0.43 \\
nucleus accumbens & $37.27 \pm 3.04$ & 0.72 \\
olfactory cortex & $68.37 \pm 2.92$ & 0.24 \\
\hline
\end{tabular}


Table 6. Rates of incorporation of $L-\left[{ }^{4} C\right]$ tryptophan into serotonin $\left(K^{C p}\right)$ for rats treated with probenecid and regional lumped constants; all values $\pm S . D ., n=15$ data points in regression

\begin{tabular}{|l|c|c|}
\hline Structure & $K^{C_{p}}(\mathrm{pmol} / \mathrm{g} / \mathrm{min})$ & Regional LC \\
\hline raphe obscurus & $64.30 \pm 5.75$ & 0.48 \\
inferior olive & $91.64 \pm 6.61$ & 0.15 \\
raphe pallidus & $65.48 \pm 5.57$ & 0.35 \\
raphe magnus & $95.87 \pm 4.75$ & 0.21 \\
locus coeruleus & $69.49 \pm 8.28$ & 0.27 \\
visual cortex & $76.31 \pm 8.37$ & 0.16 \\
pontine raphe & $82.67 \pm 4.38$ & 0.40 \\
dorsal raphe (ventromedial) & $191.29 \pm 7.31$ & 0.47 \\
dorsal raphe (all) & $166.40 \pm 6.83$ & 0.34 \\
medial raphe & $152.78 \pm 7.50$ & 0.41 \\
superior colliculus & $70.83 \pm 6.95$ & 0.21 \\
ventral tegmental area & $68.75 \pm 7.43$ & 0.28 \\
medial geniculate nucleus & $96.18 \pm 7.92$ & 0.19 \\
substantia nigra & $66.80 \pm 6.16$ & 0.25 \\
dorsal hippocampus & $64.23 \pm 7.18$ & 0.33 \\
ventral hippocampus & $98.58 \pm 8.82$ & 0.20 \\
hippocampus - CA3 & $73.23 \pm 6.81$ & 0.30 \\
\hline
\end{tabular}


Table 6 (continued). Rates of incorporation of $L-\left[{ }^{14} C\right]$-tryptophan into serotonin $\left(K^{C p}\right)$ for rats treated with probenecid and regional lumped constants; all values $\pm S . D ., n=15$ data points in regression

\begin{tabular}{|l|c|c|}
\hline Structure & $K^{C p}(\mathrm{pmol} / \mathrm{g} / \mathrm{min})$ & Regional LC \\
\hline auditory cortex & $74.11 \pm 8.40$ & 0.22 \\
lateral geniculate nucleus & $63.35 \pm 5.87$ & 0.28 \\
medial forebrain bundle & $69.11 \pm 6.38$ & 0.24 \\
ventral thalamus & $51.70 \pm 6.47$ & 0.31 \\
hypothalamus & $41.17 \pm 7.53$ & 0.43 \\
parietal cortex & $42.32 \pm 7.49$ & 0.32 \\
sensory-motor cortex & $41.96 \pm 7.43$ & 0.29 \\
caudate - medial & $46.31 \pm 7.49$ & 0.43 \\
caudate - lateral & $57.64 \pm 5.83$ & 0.31 \\
nucleus accumbens & $70.07 \pm 6.34$ & 0.38 \\
olfactory cortex & $71.90 \pm 7.14$ & 0.22 \\
\hline
\end{tabular}




\subsubsection{Determination of the Global Lumped Constant}

In this section I have tabulated the results for the regional lumped constants determined for both the probenecid-treated and control rats. These results are shown in table $7 \mathrm{a}$, and the statistics associated with the LC values are tabulated in section 7b. In addition, I have tabulated a "normalized" LC for probenecid-treated rats. This was calculated by using the average plasma tryptophan concentration for the untreated rats along with the $K^{*}$ values for the probenecid-treated rats to get the $K^{C p}$ values for the probenecid-treated rats and then calculating the regional LCs as usual. These "normalized" regional LCs may actually be more representative of the true LC for probenecid-treated rats than the non-normalized ones, for the following reason. Probenecid increases plasma free tryptophan levels significantly (table 4). This is reflected in higher $K^{C p}$ values on average for the probenecid-treated rats, which are the denominators of the equation for the LC (equation 2.19). The numerators are the $K^{C_{p}}$ values for rats in which $\alpha$ - $\left[{ }^{14} C\right]$ methyl-L-tryptophan was the tracer. These rats were not treated with probenecid, so their plasma tryptophan levels were similar to those of the untreated rats in which L- $\left[{ }^{14} C\right]$-tryptophan was used as a tracer. Thus it is reasonable to correct this difference before calculating the regional LCs. A better approach would have been to take the denominator $K^{C p}$ values from rats treated with probenecid (with $\alpha-\left[{ }^{14} C\right]$ methyl-L-tryptophan used as a tracer, naturally), but this was not done. We see from table $\mathrm{7b}$ that the non-normalized mean LC of the probenecid-treated group is significantly different from the mean LC of the control group (two-tailed t-test, $\mathrm{p}<0.05$ ), whereas the normalized mean LC of the probenecid-treated group is not significantly different ( $p>0.10$ ). In addition, I computed the skewness (G1) and kurtosis (G2) of the distribution of LC values for all three groups as well as the standard errors of G1 and G2 by standard methods [52]. These results indicate that the distribution of $\mathrm{LC}$ values in all three cases does 
not deviate significantly from a normal distribution since the skewness and kurtosis are not significantly different from zero. Since the LC values for different structures are normally distributed our assumption that they represent a statistical sampling of a single biological parameter is consistent with the data.

Based on these results, it is reasonable to use the value of 0.40 as the best estimate of the global lumped constant for the $\alpha-\left[{ }^{14} C\right]$ methyl-L-tryptophan method. 
Table 7a. Regional lumped constants for untreated and probenecid-treated rats; - pro $=$ untreated rats $;+$ pro $=$ probenecid-treated rats $;+$ pro, norm $=$ probenecid-treated rats, plasma trp levels normalized to same levels as untreated rats; $N A=$ not available

\begin{tabular}{|l|c|c|c|}
\hline Structure & LC (-pro) & LC (+pro) & LC (+pro, norm) \\
\hline raphe obscurus & 0.53 & 0.48 & 0.76 \\
inferior olive & 0.22 & 0.15 & 0.24 \\
raphe pallidus & 0.56 & 0.35 & 0.56 \\
raphe magnus & 0.38 & 0.21 & 0.33 \\
locus coeruleus & 0.49 & 0.27 & 0.43 \\
inferior colliculus & 0.18 & NA & NA \\
visual cortex & 0.18 & 0.16 & 0.25 \\
pontine raphe & 0.49 & 0.40 & 0.64 \\
superior colliculus & 0.19 & 0.47 & 0.75 \\
ventral tegmental area & 0.56 & 0.34 & 0.54 \\
medial geniculate nucleus & 0.40 & 0.41 & 0.65 \\
substantia nigra & 0.40 & 0.21 & 0.33 \\
dorsal hippocampus & 0.72 & 0.28 & 0.45 \\
ventral hippocampus & 0.42 & 0.19 & 0.30 \\
hippocampus - CA3 & 0.50 & 0.25 & 0.40 \\
auditory cortex & 0.38 & 0.22 & 0.35 \\
lateral geniculate nucleus & 0.34 & 0.28 & 0.45 \\
medial forebrain bundle & 0.33 & 0.24 & 0.38 \\
ventral thalamus & 0.28 & 0.31 & 0.49 \\
hypothalamus & 0.40 & 0.43 & 0.68 \\
parietal cortex & 0.26 & 0.32 & 0.51 \\
sensory-motor cortex & 0.30 & 0.29 & 0.46 \\
caudate - medial & 0.52 & 0.43 & 0.68 \\
caudate - lateral & 0.43 & 0.31 & 0.49 \\
nucleus accumbens & 0.72 & 0.38 & 0.60 \\
olfactory cortex & 0.24 & 0.22 & 0.35 \\
\hline
\end{tabular}


Table 7b. Statistics of Regional Lumped Constant values; abbreviations as in table 7a. Means of untreated and probenecid-treated group compared with two-tailed t-test; $N S=$ not significant

\begin{tabular}{|l|c|c|c|}
\hline & untreated & +pro & +pro (norm) \\
\cline { 2 - 4 } $\mathrm{N}$ & 26 & 25 & 25 \\
Minimum & 0.18 & 0.15 & 0.24 \\
Maximum & 0.72 & 0.48 & 0.76 \\
Mean & 0.40 & $0.29(\mathrm{p}<0.05)$ & 0.48 (NS) \\
Variance & 0.023 & 0.008 & 0.023 \\
Std. Dev. & 0.150 & 0.088 & 0.152 \\
Std. Error & 0.029 & 0.018 & 0.030 \\
C.V. & $37.4 \%$ & $30.5 \%$ & $31.5 \%$ \\
Skewness (G1) & 0.410 & 0.272 & 0.256 \\
S.E. of G1 & 0.456 & 0.464 & 0.464 \\
Kurtosis (G2) & 0.162 & -0.481 & -0.481 \\
S.E. of G2 & 0.887 & 0.902 & 0.902 \\
\hline
\end{tabular}




\subsection{Results for rates of tryptophan incorporation into protein}

\subsubsection{Sample time-activity plots}

In this section I include time-activity plots of the results for tryptophan incorporation into protein. As in the case of the serotonin synthesis data, the dark line is the regression line whose slope represents the best fit to the data. The $C_{I}^{*}(T)$ values for these experiments were calculated by using brain slices which had been washed in $20 \%$ TCA three times to remove non-protein-bound radioactivity as described in Materials and Methods. Since one would expect that this would remove the precursor pool (from the measurements only, i.e. the $C_{I}^{*}(T)$ value measured would not reflect the combined precursor pool and labelled protein but only labelled protein, according to the model described in the Theory section), one would expect that the time-activity plots should have only a linear region representing the irreversible uptake of label into protein. This indeed appears to be the case for the probenecid-treated rats but less so for the controls. This will be discussed further in the Discussion section. 


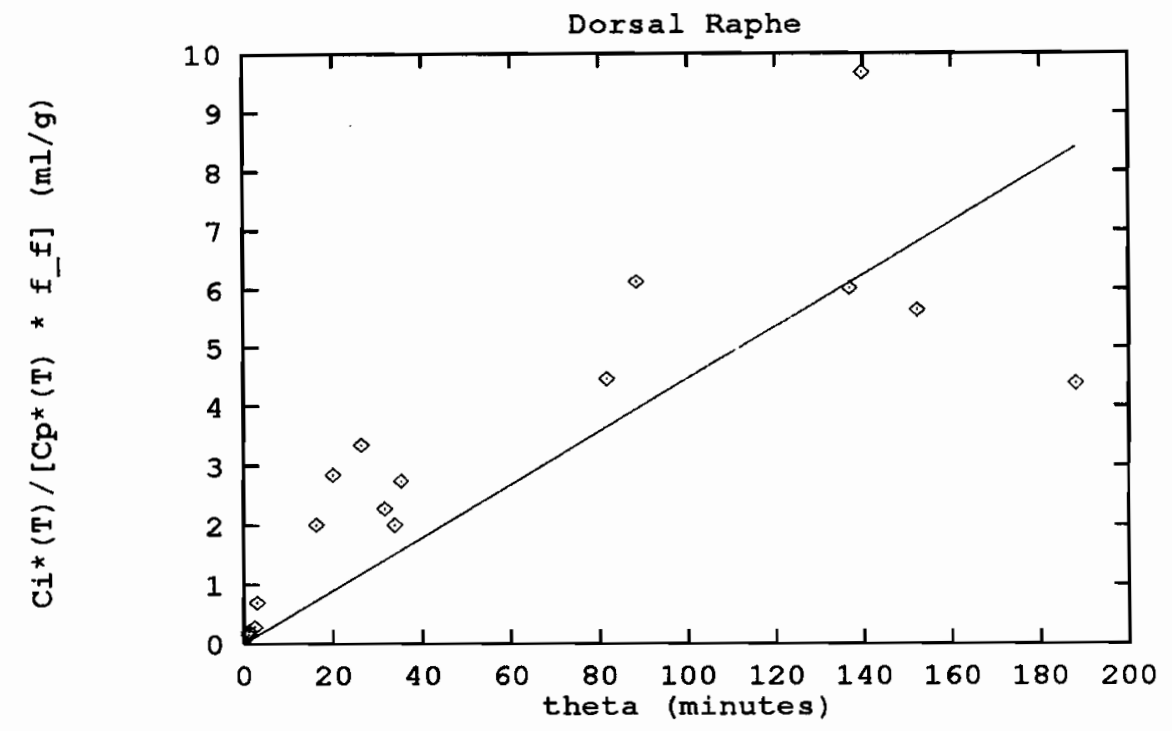

Figure 4.8: Time-activity plot for protein synthesis for rats not treated with probenecid (dorsal raphe nucleus).

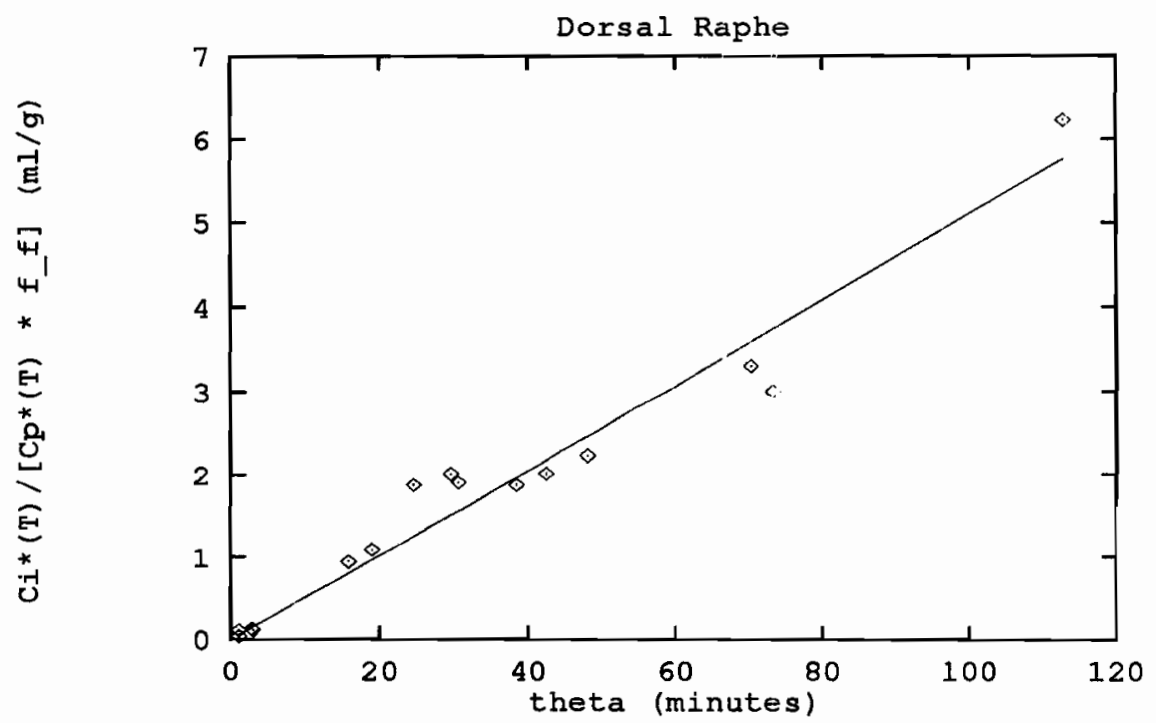

Figure 4.9: Time-activity plot for protein synthesis for rats treated with probenecid (dorsal raphe nucleus) 


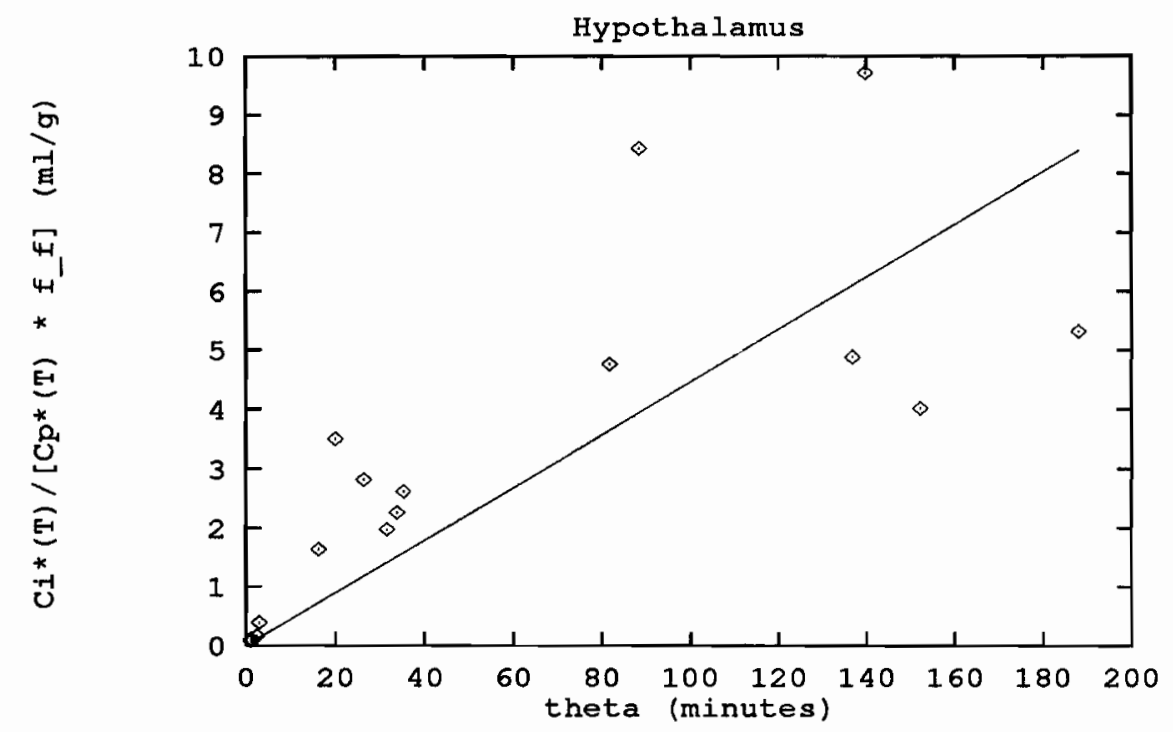

Figure 4.10: Time-activity plot for protein synthesis for rats not treated with probenecid (hypothalamus).

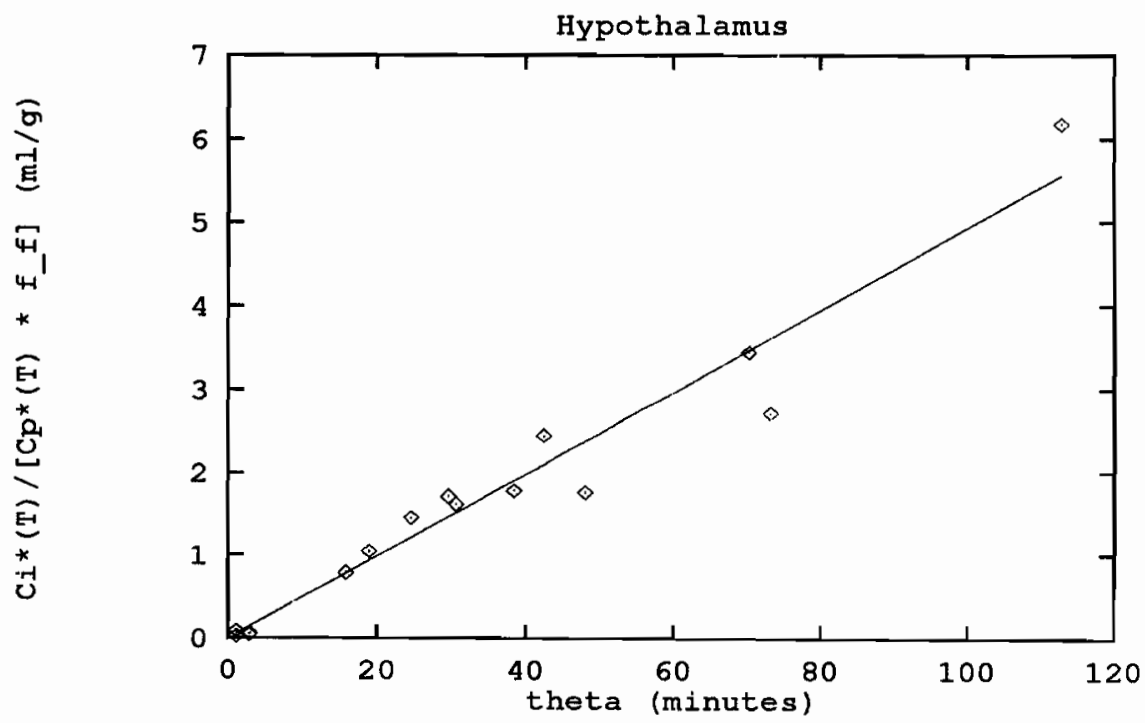

Figure 4.11: Time-activity plot for protein synthesis for rats treated with probenecid (hypothalamus) 


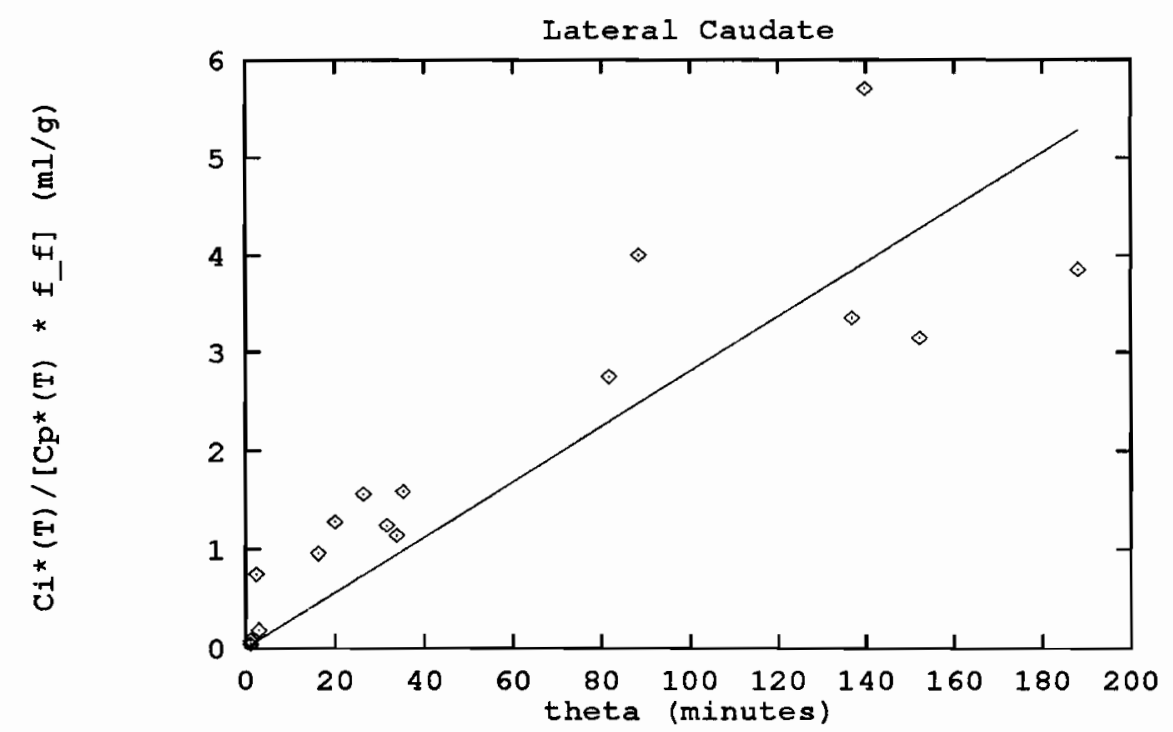

Figure 4.12: Time-activity plot for protein synthesis for rats not treated with probenecid (lateral caudate nucleus).

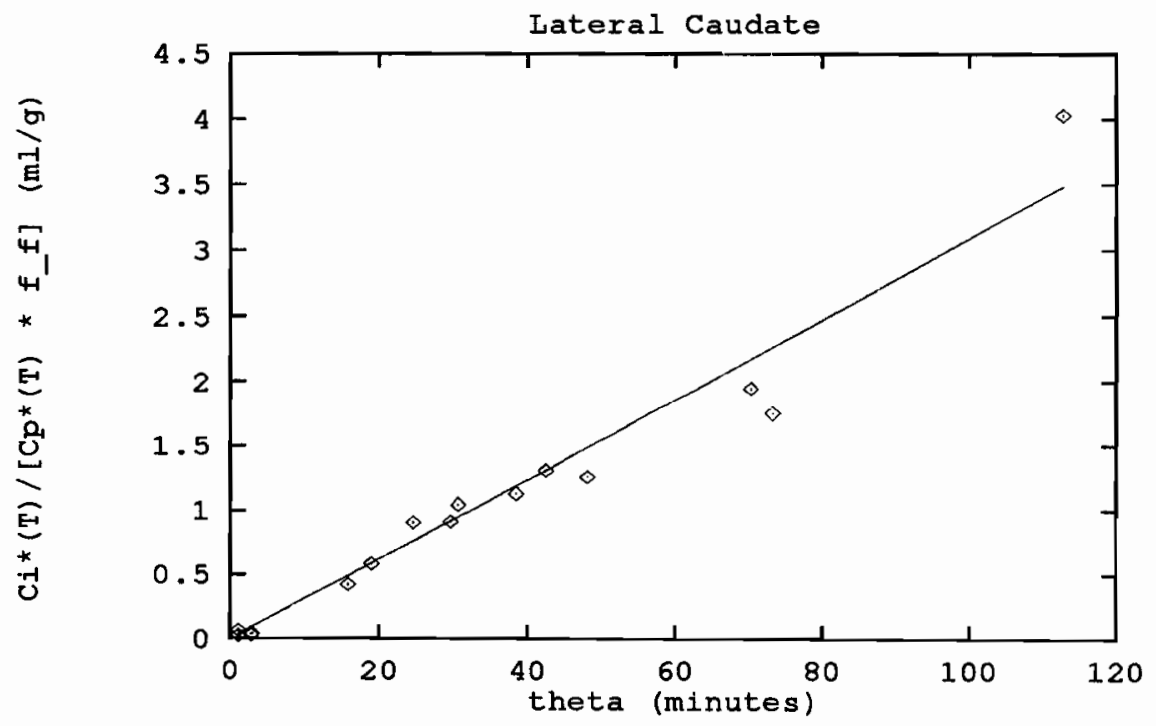

Figure 4.13: Time-activity plot for protein synthesis for rats treated with probenecid (lateral caudate nucleus) 


\subsubsection{Rates of Tryptophan Incorporation into Protein}

In table 8a I have tabulated the results for tryptophan incorporation into protein. Since the $K^{C p}$ values (which equal the rate of tryptophan incorporation into brain) are obtained by multiplying the $K^{*}$ values by the concentration of free tryptophan $\left(C_{p}\right)$, and since $C_{p}$ is much higher in probenecid-treated rats, the rate of tryptophan incorporation into brain is consequently much higher in probenecid-treated versus control rats. In order to dissociate this effect, i.e. to see if probenecid has any effect on the rate of tryptophan incorporation into protein aside from its effect on plasma free tryptophan, a "normalized" rate of tryptophan incorporation into proteins was also calculated by using the plasma concentration of free tryptophan for the controls to calculate $K^{C p}$ for both controls and the treatment group. Since this normalized rate does not reflect the true rate of tryptophan incorporation into protein for probenecidtreated rats, no standard error was calculated. These values can be directly compared with the results for control rats to determine the effect of probenecid on tryptophan incorporation into proteins above and beyond its effect on plasma tryptophan levels. This ratio is tabulated below, and reflects the effect of probenecid on the $K^{*}$ values; i.e. the change in the unidirectional rate of uptake of tracer into the brain caused by the tracer.

In table $8 \mathrm{~b}$ I have tabulated the statistics on the ratios of the (normalized) effect of probenecid on the rate of tryptophan incorporation into protein to the rate in controls. Probenecid caused this rate to increase between 3 and $36 \%$ (average 17\%). The skewness and kurtosis (G1 and G2) are not significantly different from zero ( $p>0.10$, two-tailed t-test), so the distribution of values is not significantly different from a normal distribution. Thus, the data indicates that probenecid increases the rate of incorporation of exogenous tryptophan (i.e. plasma-derived, as opposed to that derived from protein degradation, for example) into brain proteins by $17 \%$. 
Since the data is approximately normally distributed, it appears that probenecid has a comparable effect on the rate of tryptophan incorporation into proteins throughout the brain and the values tabulated represent estimates of this value sampled from a normal distribution. 
Table 8a. Rates of incorporation of $\left.L-\rho^{4} C\right]$-tryptophan into protein $\left(K^{C p}\right.$, pmol/g/min) for untreated rats and probenecid-treated rats; also normalized values for probenecid (see text); all values \pm S.E., $n=16$ data points in regression

\begin{tabular}{|l|c|c|c|c|}
\hline Structure & - Pro & + Pro & + Pro, norm & + Pro/- Pro \\
\hline raphe obscurus & $255.17 \pm 7.44$ & $448.92 \pm 5.16$ & 299.91 & 1.18 \\
inferior olive & $355.63 \pm 10.88$ & $651.45 \pm 7.43$ & 435.22 & 1.22 \\
raphe pallidus & $299.08 \pm 9.44$ & $501.04 \pm 6.45$ & 334.73 & 1.12 \\
cerebellum(gray) & $402.99 \pm 14.88$ & $770.39 \pm 6.91$ & 514.68 & 1.28 \\
raphe magnus & $261.56 \pm 8.96$ & $530.96 \pm 5.78$ & 354.72 & 1.36 \\
locus coeruleus & $286.52 \pm 9.04$ & $517.03 \pm 5.73$ & 345.42 & 1.21 \\
inferior colliculus & $344.99 \pm 12.56$ & $617.96 \pm 6.40$ & 412.84 & 1.20 \\
visual cortex & $302.44 \pm 9.60$ & $564.71 \pm 6.14$ & 377.27 & 1.25 \\
pontine raphe & $286.04 \pm 8.64$ & $493.81 \pm 5.68$ & 329.90 & 1.15 \\
dorsal raphe (ventromed.) & $431.30 \pm 15.36$ & $806.51 \pm 9.44$ & 538.81 & 1.25 \\
dorsal raphe (all) & $370.43 \pm 12.56$ & $634.68 \pm 6.71$ & 424.01 & 1.14 \\
medial raphe & $324.04 \pm 11.20$ & $590.30 \pm 8.00$ & 394.37 & 1.22 \\
superior colliculus & $283.16 \pm 8.80$ & $477.30 \pm 6.66$ & 318.87 & 1.13 \\
ventral tegmental area & $199.97 \pm 4.00$ & $307.02 \pm 4.90$ & 205.11 & 1.03 \\
medial geniculate nucleus & $311.72 \pm 9.52$ & $509.55 \pm 4.43$ & 340.42 & 1.09 \\
substantia nigra & $213.57 \pm 3.92$ & $344.28 \pm 3.66$ & 230.00 & 1.08 \\
dorsal hippocampus & $392.75 \pm 10.88$ & $652.22 \pm 7.48$ & 435.73 & 1.11 \\
ventral hippocampus & $471.14 \pm 12.96$ & $795.88 \pm 11.77$ & 531.71 & 1.13 \\
hippocampus - CA3 & $572.08 \pm 16.07$ & $1083.45 \pm 11.40$ & 723.82 & 1.27 \\
\hline
\end{tabular}


Table 8a (continued). Rates of incorporation of $L-\left[{ }^{4} C\right]$-tryptophan into protein $\left(K^{C p}, n m o l / g / m i n ~ \times 10^{3}\right)$ for untreated rats and probenecid-treated rats; also normalized values for probenecid (see text); all values $\pm S . E ., n=16$ data points in regression

\begin{tabular}{|l|c|c|c|c|}
\hline Structure & - Pro & + Pro & + Pro, norm & + Pro/(- Pro) \\
\hline auditory cortex & $324.76 \pm 8.64$ & $565.02 \pm 6.13$ & 377.48 & 1.16 \\
dorsal thalamus & $263.40 \pm 6.88$ & $449.69 \pm 6.14$ & 300.43 & 1.14 \\
lateral geniculate nucleus & $278.04 \pm 7.68$ & $448.77 \pm 6.45$ & 299.81 & 1.08 \\
medial forebrain bundle & $243.89 \pm 6.24$ & $433.96 \pm 0.88$ & 289.91 & 1.19 \\
ventral thalamus & $273.40 \pm 6.00$ & $444.79 \pm 7.17$ & 297.15 & 1.09 \\
hypothalamus & $369.87 \pm 13.52$ & $611.98 \pm 7.28$ & 408.85 & 1.11 \\
parietal cortex & $325.48 \pm 9.84$ & $559.86 \pm 6.35$ & 374.03 & 1.15 \\
sensory-motor cortex & $326.92 \pm 8.96$ & $579.98 \pm 6.14$ & 387.47 & 1.19 \\
caudate- medial & $228.13 \pm 5.36$ & $379.26 \pm 5.99$ & 253.37 & 1.11 \\
caudate - lateral & $232.77 \pm 5.68$ & $383.39 \pm 4.85$ & 256.13 & 1.10 \\
nucleus accumbens & $225.81 \pm 4.96$ & $366.21 \pm 4.90$ & 244.65 & 1.08 \\
olfactory cortex & $502.81 \pm 17.76$ & $984.01 \pm 12.90$ & 657.39 & 1.31 \\
frontal cortex & $323.56 \pm 9.52$ & $579.47 \pm 6.71$ & 387.13 & 1.20 \\
\hline
\end{tabular}


Table 8b. Statistics of Effect of Probenecid Treatment on Regional Rates of Tryptophan Incorporation into Brain Proteins.

\begin{tabular}{|l|c|}
\hline $\mathrm{N}$ & 32 \\
Minimum & 1.03 \\
Maximum & 1.36 \\
Mean & 1.17 \\
Variance & 0.006 \\
Std. Dev. & 0.075 \\
Std. Error & 0.013 \\
C.V. & $6.43 \%$ \\
Skewness (G1) & 0.645 \\
S.E. of G1 & 0.414 \\
Kurtosis (G2) & 0.223 \\
S.E. of G2 & 0.809 \\
\hline
\end{tabular}




\section{Chapter 5}

\section{Discussion}

\subsection{Determination of the lumped constant}

In the previous sections I have described the method we have used to estimate the lumped constant (LC) for the $\alpha$-methyl-tryptophan method for measuring the local rates of 5 -HT synthesis in the rat brain. The $\alpha$-methyl-tryptophan method uses the compound $\alpha$-[14 $C]$-methyl-L-tryptophan, a radioactive tryptophan analogue, as a metabolic tracer for the pathway of 5 -HT synthesis for the following reasons:

1. It is transported into the brain tissue and undergoes hydroxylation by tryptophan hydroxylase which after decarboxylation yields $\alpha-\left[{ }^{14} C\right]$-methyl-5-HT;

2. $\alpha$-methyl-5-HT is not a substrate for monoamine oxidase (MAO) degradation to any significant extent, so that it accumulates irreversibly in the brain tissue, permitting quantification via quantitative autoradiography.

3. It is not incorporated into proteins to any significant extent, thus simplifying the kinetic model. 
These facts enable us to model the system with an irreversible compartment into which tracer moves and is trapped in the brain.

The kinetic model described in the Theory section provides a means of estimating $K^{*}$, the net unidirectional influx constant, for $\alpha$-methyl-tryptophan incorporation into $\alpha$-methyl-5-HT. If this constant were equal to the $K^{*}$ for tryptophan entry into the 5-HT/5HIAA compartments, the rate of 5 -HT synthesis would equal $K^{*}$ times $C_{p}$, the plasma free tryptophan concentration (this quantity is referred to in the Theory section as $f_{f} \cdot C_{p}$ i.e. the free fraction times the total plasma tryptophan; I refer to the free tryptophan as $C_{p}$ here for the sake of brevity). However, since the kinetic parameters (e.g. $K_{M}$ and $V_{m a x}$ ) of the rate-limiting enzyme in 5-HT synthesis, tryptophan hydroxylase (TH) are not necessarily the same for tryptophan and $\alpha$ methyl-tryptophan, we must have some way of quantifying and compensating for this difference. Sokoloff's paper [21] introduced the concept of the "lumped constant" (LC) as a way of correcting for the difference in the kinetics of metabolism of the tracer and the tracee (substance whose metabolism is being traced). The method I have used here to determine the lumped constant is to calculate the rate of 5 - $\mathrm{HT}$ synthesis $\left(K^{C p}\right)$ in rats using $\left[{ }^{14} C\right]$-L-tryptophan as a tracer instead of $\alpha$ - $\left[{ }^{14} C\right]$-methylL-tryptophan. The $K^{C p}$ values for $\alpha$-[14 $\left.C\right]$-methyl-L-tryptophan have previously been computed [34]. Given this information, the LC can be estimated as described in the Theory section:

$$
L C=\frac{K^{C_{p}}(\alpha-\text { methyl }-L-\text { tryptophan })}{K^{C_{p}}(L-\text { tryptophan })}
$$

This method gives regional LC values which can be averaged to give an estimate for the global LC; the validity of this is discussed further below. Our estimate of the LC was $0.40 \pm 0.15$ for normal rats ( \pm S.D., $n=26$ ). In addition, I performed experiments on rats treated with the drug probenecid, which blocks the efflux of 5- 
HIAA, a 5-HT metabolite, from rat brain. This allowed the use of a simpler and potentially more accurate method (the "linearized time-activity method" described in the Theory section) to determine $K^{*}$ as compared with the approach used with untreated rats, where the loss of 5-HIAA from the tissue during the course of the experiments had to be explicitly modelled. However, this approach had the limitation that the same $\alpha$-methyl-tryptophan data was used to calculate the LC with untreated and probenecid-treated rats; this data derived from untreated rats as well so that the validity of using it to calculate an $\mathrm{LC}$ with probenecid-treated rats can be questioned. For example, it is conceivable that probenecid treatment would have a differential effect on the uptake of tryptophan and $\alpha$-methyl-tryptophan, although my data argues against this (see table $7 \mathrm{~b}$ and below). In fact, it was found that in this case the LC was estimated to be equal to $0.29 \pm 0.09$ ( \pm S.D., $n=26$ ), which is significantly different from the $\mathrm{LC}$ for untreated rats. This was unexpected, since unless probenecid treatment directly affected the kinetic parameters of the rate limiting enzyme TH, one would not expect any change in the lumped constant. However, we noticed that one effect of probenecid treatment was to increase the plasma free tryptophan levels from $7.56 \mathrm{nmol} / \mathrm{ml}$ to $12.02 \mathrm{nmol} / \mathrm{ml}$. Even if the $K^{*}$ values for untreated and probenecid-treated rats were identical, the difference in free tryptophan levels would increase the $K^{C p}$ for treated rats and thus depress the apparent LC. This may not reflect a true change in the $\mathrm{LC}$ for probenecid-treated rats, however. If the rats in which $\alpha-\left[{ }^{14} C\right]$-methyl-L-tryptophan was used as a tracer had been treated with probenecid, they might also have shown the large increase in plasma free tryptophan, and thus both the numerator and denominator of equation (5.1) would be increased by comparable amounts, so that the LC would not be significantly different. The best way to check this possibility is to perform experiments using $\alpha-\left[{ }^{14} \mathrm{C}\right]$-methyl-Ltryptophan as a tracer on probenecid-treated rats. These experiments are now in progress in our laboratory. Nevertheless, we can get at least a rough idea of the 
effect of the change in free tryptophan concentrations on the LC by "normalizing" for free tryptophan concentrations as follows: the $K^{C p}$ values for the probenecidtreated rats were recomputed using the $C_{p}$ values for the untreated rats. Then the $\mathrm{LC}$ was recalculated. In this case, the $\mathrm{LC}$ was estimated to be $0.48 \pm 0.15(n=26, \pm$ S.D.), which was not significantly different from the value with untreated rats. This provides evidence that the main effect of probenecid on the rate of 5-HT synthesis is to increase plasma free tryptophan concentrations, and that probenecid causes no significant change in $K^{*}$ values. Nevertheless, I have not averaged the LC for untreated and probenecid-treated rats, since to get a reasonable estimate of the LC for probenecid-treated rats requires us to perform experiments using $\alpha-\left[{ }^{14} C\right]$-methylL-tryptophan as a tracer in probenecid-treated rats.

This thesis represents the first time the lumped constant for this method has been explicitly calculated from experimental data. In a previous paper [19] the LC was estimated by using published values of $K_{M}$ 's and $V_{M A X}$ 's for tryptophan hydroxylase, accumulated from several laboratories, to give the LC according to equation (2.5). The estimated LC in that paper was 0.46. This is well within the error range of our estimate, so the values for the local rates of 5-HT synthesis given in that paper are consistent with our results and can be considered reasonable estimates of the local rates of 5-HT synthesis. In this thesis I have also demonstrated (see below) that the distribution of LC values throughout the brain is not significantly different from a normal distribution, which indicates that the LC is probably uniform throughout rat brain.

The regional LC values were averaged to give an estimate of the global LC. This could be criticized on the grounds that the regional variations in the $\mathrm{LC}$ are real and do not simply represent statistical fluctuations. I believe that this is not the case for the following reasons. The dispersion statistics G1 and G2 (skewness and kurtosis) were calculated for the ensemble of LC values. Neither statistic showed a statistically 
significant difference from zero, the value which would be obtained from a pure normal distribution. Thus, there is no statistically significant bimodality or multimodality in the $\mathrm{LC}$ values we have obtained, supporting the notion that the measured $\mathrm{LC}$ values are statistical fluctuations from a "true" LC value. Furthermore, if regional differences in $\mathrm{LC}$ values were shown to be statistically significant, it would be necessary to explain this variation. It is possible that various isoforms of tryptophan hydroxylase exist, but it seems unlikely that the variations in the enzyme would be such that the enzyme's relative affinity for $\alpha$-methyl-tryptophan relative to tryptophan would be significantly changed, especially since the active sites would probably be the same.

As mentioned above, my results indicate that probenecid treatment causes a large increase in plasma free tryptophan concentration. The most probable explanation of this is that probenecid displaces tryptophan from binding sites on serum albumin, which normally binds $80-90 \%$ of the plasma tryptophan. Probenecid has been shown to cause an increase in the free fractions of several drugs which bind to plasma albumin, among them indomethacin [53], methotrexate [54] and ceftriaxone [55]. Thus it appears that probenecid has a high affinity for the same binding sites on plasma albumin that bind the above compounds and can displace them if present at high enough concentrations. It is interesting to note that the total tryptophan concentration in the plasma is reduced by probenecid despite the large increase in free tryptophan levels, which suggests that probenecid at the concentration I used displaces tryptophan from plasma albumin very strongly. Probenecid treatment also caused a number of other changes in plasma amino acid levels, summarized in table 4. Most significantly, the apparent $K_{M}$ for tryptophan transport is reduced by probenecid treatment by about $20 \%$. This would imply that transport of tryptophan across the BBB is favoured in probenecid-treated rats relative to control rats. However, since tryptophan transport across the BBB is not normally the rate-limiting step in 5-HT synthesis, this may have little effect on the rate of 5-HT synthesis. In addition, the PCR for free tryp- 
tophan (but not total tryptophan) was significantly increased, indicating a greater preference of the LNAA transporter for free tryptophan, as a result of the higher free tryptophan levels.

Probenecid also had the effect of increasing the $\mathrm{pO}_{2}$ in plasma. To the best of our knowledge, this effect has not been reported before, and the reason for it is unclear. It is possible that probenecid acts to displace oxygen from the hemoglobin molecule, but such speculations have no experimental support as yet. Probenecid causes a statistically significant rise in the $\mathrm{pO}_{2}$ by about $25 \%$ of normal levels. It is well known that the rate of 5 -HT synthesis can be increased by increasing the oxygen concentration of the blood (e.g. $[29,56,57])$. However, as mentioned, once the difference in plasma free tryptophan levels was accounted for, there was little difference in the rate of $5-\mathrm{HT}$ synthesis with or without probenecid (in other words, the $K^{*}$ values for probenecid-treated and untreated rats were comparable) suggesting that a $25 \%$ increase in oxygen concentration in this range of $\mathrm{pO}_{2}$ values is not sufficient to cause an appreciable increase in the rate of 5-HT synthesis. This is in agreement with data reported earlier in the rat [57].

It is also worth discussing why it is desirable to calculate the lumped constant in the first place. If we are only interested in the relative rates of 5-HT synthesis in different brain regions in particular physiological states, or in the same region in different physiological states, then the lumped constant is unnecessary since only relative values of the rates of 5 -HT synthesis are needed. However, if we want to compare the rate of 5-HT synthesis with that of other neurotransmitters, for instance, it is useful to have an absolute measure since this will permit the assessment of the relative activities of different neurotransmitter systems.

Based on our data, therefore, I estimate the lumped constant for the $\alpha$-methyltryptophan method to be $0.40 \pm 0.15$. 


\subsection{Determination of the rate of tryptophan in- corporation into protein}

In this thesis $I$ have also calculated the rate of tryptophan incorporation into brain proteins, for both normal and probenecid-treated rats. This was not a primary goal of the work done, but the data accumulated in the course of estimating the lumped constant made it possible to calculate tryptophan incorporation into proteins without performing any new experiments, so the data is included here. It should be noted that the rates of tryptophan incorporation into protein refer only to exogenous tryptophan (tryptophan from the plasma) since it is the net unidirectional influx rate of plasma tryptophan into the protein pool which we have calculated. Endogenous brain tryptophan is also incorporated into proteins; therefore the total rate of tryptophan incorporation into protein may be greater than the figures given in table $8 \mathrm{a}$.

The kinetic model used for calculating tryptophan incorporation into protein is the "linearized time-activity method" described in the Theory section. As mentioned in the Materials and Methods section, an autoradiogram of a brain slice from a rat which has been injected with $\left[{ }^{14} C\right]$-L-tryptophan consists of numerous radiolabelled components: the precursor tryptophan pool (or pools); 5-HT; 5-HIAA; and radioactive tryptophan incorporated into proteins. The kinetic model used for the determination of $K^{*}$ postulates only one irreversible compartment, whereas in this case we have two distinct irreversible compartments. If only the total brain radioactivity is measured it is not possible to mathematically distinguish the rate of tracer influx into the two compartments. In order to deal with this, I prepared replicate slices for all brain regions (adjacent slices while cutting the brain) and treated the two sets of slices as follows. The first set was directly exposed to x-ray film along with calibrated radioactivity standards to give the total radioactivities in various brain regions. The other set of 
slices were washed in trichloroacetic acid (TCA); this procedure precipitates proteins but washes away non-protein-bound radioactivity. Autoradiography of these brain slices gives the total protein-bound radioactivities in various brain regions. For the 5HT synthesis calculations, the protein-bound radioactivities were subtracted from the total radioactivities to give the non-protein-bound radioactivities which could be used as input to the kinetic models discussed previously to give the $K^{*}$ for (exogenous) tryptophan conversion into serotonin. I also used the protein-bound radioactivity data to calculate the $K^{*}$ for tryptophan incorporation into protein. Since the precursor pool for this process (which presumably was part of the larger precursor pool of tryptophan, although sub-compartmentation of intracellular tryptophan for various metabolic pathways is a possibility) would probably have been washed away by TCA treatment, we would expect that the tissue volume of distribution of the tracer $\left(\frac{C_{I}^{*}(T)}{C_{p}^{*}(T) \cdot f_{f}}\right.$ in equation $\left.(2.9)\right)$ would be a linear function of the exposure time $(\Theta(T))$ with y-intercept zero. The y-intercept represents the apparent steady-state volume of distribution of the tracee, which in this case must be zero since the precursor pool was washed away by TCA-washing before $C_{I}^{*}(T)$ was calculated for these autoradiograms. Stated differently, the tracer should accumulate linearly with the exposure time in the brain tissue. This indeed appears to be the case for probenecid-treated rats, as indicated by figures $4.9,4.11$ and 4.13 in the Results section. The results for normal rats are less clear. There is a great deal of scatter in the data, which I attribute to the fact that these experiments, especially those corresponding to the rightmost points of figures $4.8,4.10$, and 4.12 were among the earliest experiments I performed, and my experimental technique was not as good as in the later experiments involving probenecid-treated rats.

Looking at the results in table 8 , we can see that the rate of tryptophan incorporation into proteins for normal rats ranges from $199.97 \mathrm{pmol} / \mathrm{g} / \mathrm{min}$ (for the ventral tegmental area) to $572.08 \mathrm{pmol} / \mathrm{g} / \mathrm{min}$ (for the CA3 region of the hippocampus). 
Probenecid treatment appears to cause a sharp increase in the rate of tryptophan incorporation into protein. However, most of this increase can be attributed to the rise in plasma free tryptophan concentration, since the rate was calculated by multiplying this with $K^{*}$. If we "normalize" this effect by recalculating the rate of tryptophan incorporation into protein for probenecid-treated rats assuming the same free tryptophan levels as in normal rats, the differences in the levels of tryptophan incorporation into protein between normal and probenecid-treated rats is greatly reduced. As shown in table 8 , this effect is an increase of $17 \%$ on average.

The estimation of the rate of tryptophan incoporation into brain proteins does not appear to have been done before. However, the rates of incorporation of other amino acids into brain protein have been measured [58, 59, 60,61], using various radiolabelled amino acids (i.e. valine, leucine, methionine) as tracers. For example, Kirikae et. al. [58] estimated the rate of incorporation of valine into proteins. Their estimates average $2.86 \mathrm{nmol} / \mathrm{g} / \mathrm{min}$ for gray matter structures, which is about 6-10 times our estimates for tryptophan. Smith [59] estimated the local rates of leucine incorporation into protein at between 9 and $12 \mathrm{nmol} / \mathrm{g} / \mathrm{min}$ for different brain structures. This rate comprised the total rate of leucine incorporation into protein, and not just the rate of incorporation of exogenous leucine, unlike the procedures I have described. Keen et. al. [61] estimated a value of $3.2 \mathrm{nmol} / \mathrm{g} / \mathrm{min}$ for incorporation of exogenous leucine into rat brain protein, while Lestage et. al. [60] estimated values in the range of 0.72 to $3.08 \mathrm{nmol} / \mathrm{g} / \mathrm{min}$ for incorporation of exogenous methionine into rat brain protein. Considering the greater abundance of these amino acids in brain protein relative to tryptophan, my estimates appear to be reasonable. 


\subsection{Possible future directions}

As mentioned above, a necessary addition to the experiments described above is to use $\alpha-\left[{ }^{14} C\right]$-methyl-L-tryptophan as a tracer on probenecid-treated rats. This will permit us to correctly estimate the lumped constant for probenecid-treated rats. Based on the data given above, we would not expect the LC to deviate significantly from the value of 0.40 calculated for untreated rats.

The main use of the LC would be to estimate the absolute rates of 5 -HT synthesis in different physiological states. It would be desirable to know the LC for this method in humans as well. Human data of this kind can only be obtained through positron emission tomography (PET) studies. Therefore the procedure described above is not feasible, since one cannot remove the contribution of radiolabelled tryptophan incorporated into brain in the same way. However, a more complicated kinetic model, which takes into account the conversion of tryptophan into protein as well as into 5HT, might be used to estimate the $K^{*}$ for tryptophan conversion into 5 -HT in human subjects. Since $K^{*}$ values for $\alpha$-methyl-L-tryptophan conversion to $\alpha$-methyl-5-HT can be obtained in humans using ${ }^{11} C$-labelled $\alpha$-methyl-L-tryptophan and PET, this would enable us to calculate the LC in humans. In general, however, the more complex a kinetic model is, the less certain its parameter values are, so it is likely the $K^{*}$ values computed by this method would have large errors. In addition, a large fraction of the radioactivity in experiments using ${ }^{11} C$-labelled L-tryptophan would be in the protein pool, so that the estimate of the radioactivity not incorporated into protein would itself have large errors even before estimation of $K^{*}$. Alternately, one might also use the arterio-venous concentration difference as in Sokoloff's model (equation 2.20) to estimate the global LC.

The LC value estimated here should be of use in a wide variety of studies in the rat. In fact, the only conditions which might invalidate the use of the LC itself would be 
pharmacological manipulations which might alter the relative affinities of tryptophan and $\alpha$-methyl-L-tryptophan for the enzyme tryptophan hydroxylase. Even if the absolute affinities of $\mathrm{TH}$ for the two substrates were altered by a particular treatment, as long as the relative affinities remain the same the $\mathrm{LC}$ will not change. 


\section{Chapter 6}

\section{Conclusions}

In this thesis, I have described the method I have used to estimate the lumped constant (LC) for the $\alpha$-methyl-L-tryptophan method for determining the rate of serotonin (5-HT) synthesis in rat brain. The lumped constant was estimated to be $0.40 \pm 0.15$ ( \pm S.D., $n=26$ ). The LC was also estimated for probenecid-treated rats. This LC was estimated to be $0.29 \pm 0.09$ ( \pm S.D., $n=25$ ). However, probenecid was demonstrated to cause a large increase in the plasma free tryptophan levels. When this was corrected for, the $\mathrm{LC}$ with probenecid was estimated to be $0.48 \pm 0.15$, which is not significantly different from the LC for normal rats. This is to be expected, since drug treatments should not change the LC unless they differentially alter the relative affinities of tryptophan and $\alpha$-methyl-tryptophan for the rate-limiting enzyme for 5-HT synthesis (tryptophan hydroxylase), and there is no evidence that probenecid does this.

In addition, as a side benefit of the procedure used, I computed the rate of incorporation of tryptophan into brain proteins. This rate ranged from $199.97 \mathrm{pmol} / \mathrm{g} / \mathrm{min}$ for the ventral tegmental area to $572.08 \mathrm{pmol} / \mathrm{g} / \mathrm{min}$ for the CA3 region of the hippocampus. The rate of tryptophan incorporation into protein was also calculated for 
rats treated with probenecid. These values were considerably higher; however, most of the difference again resulted from the increase in plasma free tryptophan levels. When this effect was corrected for, probenecid was shown to cause an increase in the rate of tryptophan incorporation into brain proteins of $17 \%$ on average. 


\section{References}

[1] Jack R. Cooper, Floyd E. Bloom, and Robert H. Roth. The Biochemical Basis of Neuropharmacology, 5th ed. Oxford University Press, Oxford, 1986.

[2] J. J. Lopez-Ibor. The involvement of serotonin in psychiatric disorders and behavior. British Journal of Psychiatry, 153 (supplement 3):26-39, 1988.

[3] E. Edwards, K. Harkins, G. Wright, and Henn F. Modulation of ${ }^{3} \mathrm{H}$-paroxetine binding to the 5-hydroxytryptamine uptake site in an animal model of depression. Journal of Neurochemistry, 56(5):1581-1586, 1991.

[4] R. J. Katz. Neurobiology of obsessive-compulsive disorder - a serotonergic basis of Freudian repression. Neuroscience and Biobehavioral Reviews, 15:375-381, 1991.

[5] T. R. Insel, J. Zohar, C. Benkelfat, and D. L. Murphy. Serotonin in obsessions, compulsions, and the control of aggressive impulses. Annals of the New York Academy of Sciences, 600:574-586, 1990.

[6] T. D. Brewerton. Fluoxetine-induced suicidality, serotonin, and seasonality. $B i$ ological Psychiatry, 30(2):190-196, 1991.

[7] J. J. Mann, V. Arango, and M. D. Underwood. Serotonin and suicidal behavior. Annals of the New York Academy of Sciences, 600:476-485, 1990. 
[8] E. M. Nikulina. Neural control of predatory aggression in wild and domesticated animals. Neuroscience and Biobehavioral Reviews, 15(4):545-547, 1991.

[9] B. Olivier, J. Mos, J. Schipper, and J. Vanderheyden. Serotonin and aggressive behavior in the rat. Psychopharmacology, 89(4):S 26 - S 26, 1986.

[10] D. C. Jimerson, M. D. Lesem, A. P. Hegg, and T. D. Brewerton. Serotonin in human eating disorders. Annals of the New York Academy of Sciences, 600:532$544,1990$.

[11] K. P. Lesch, M. Wiesmann, A. Hoh, and T. Muller. 5-HT1a receptor-effector system responsivity in panic disorder. Psychopharmacology, 106(1):111-117, 1992.

[12] J. W. Lance. 5-hydroxytryptamine and its role in migraine. European Neurology, 31(5):279-281, 1991.

[13] Arora R. C., O. B. Emery, and H. Y. Meltzer. Serotonin uptake in the bloodplatelets of Alzheimer's-disease patients. Neurology, 41(8):1307-1309, 1991.

[14] A. Bleich, S. L. Brown, R. Kahn, and H. M. Vanpraag. The role of serotonin in schizophrenia. Schizophrenia bulletin, 14(2):297-315, 1988.

[15] B. E. Jones. Paradoxical sleep and its chemical/structural substrates in the brain. Neuroscience, 40(3):637-656, 1991.

[16] P. K. Eide and K. Hole. Interactions between serotonin and substance-p in the spinal regulation of nociception. Brain Research, 550(2):225-230, 1991.

[17] W. J. McEntee and T. H. Crook. Serotonin, memory, and the aging brain. Psychopharmacology, 103(2):143-149, 1991. 
[18] B. B. Gorzalka, S. D. Mendelson, and N. V. Watson. Serotonin receptor subtypes and sexual behavior. Annals of the New York Academy of Sciences, 600:435-446, 1990.

[19] M. Diksic, S. Nagahiro, T.L. Sourkes, and Y.L. Yamamoto. A new method to measure brain serotonin synthesis in vivo. I. Theory and basic data for a biological model. J. Cereb. Blood Flow and Metabolism, 10:1-12, 1990.

[20] S. Nagahiro, A. Takada, M. Diksic, T. L. Sourkes, K. Missala, and Y.L. Yamamoto. A new method to measure brain serotonin synthesis in vivo. II. A practical method tested in normal and lithium-treated rats. J. Cereb. Blood Flow and Metabolism, 10:13-21, 1990.

[21] L. Sokoloff, M. Reivich, C. Kennedy, M.H. Des Rosiers, C. S. Patlak, K. D. Pettigrew, O. Sakurada, and M. Shinohara. The $\left[{ }^{14} \mathrm{C}\right]$ deoxyglucose method for the measurement of local cerebral glucose utilization: theory, procedure, and normal values in the conscious and anaesthetized albino rat. Journal of Neurochemistry, 28:897-916, 1977.

[22] M. C. Boadle-Biber. Biosynthesis of serotonin. In N. N. Osborne, editor, Biology of Serotonergic Transmission, chapter 3, pages 63-94. J. Wiley and Sons, 1982.

[23] D.L. Bloxam and G. Curzon. A study of proposed determinants of brain tryptophan concentration in rats after portocaval anastomosis or sham operation. Journal of Neurochemistry, 31:1255-1263, 1978.

[24] G.H. Anderson. Control of protein and energy intake: role of plasma amino acids and brain neurotransmitters. Can. J. Physiol. Pharmacol., 57:1043-1057, 1979. 
[25] W. M. Pardridge. Kinetics of competitive inhibition of neutral amino acid transport across the blood-brain barrier. Journal of Neurochemistry, 28:103-108, 1977.

[26] L. P. Miller, W. M. Pardridge, L. D. Braun, and W. H. Oldendorf. Kinetic constants for blood-brain barrier amino acid transport in concious rats. Journal of Neurochemistry, 45(5):1427-1432, 1985.

[27] P. M. Herlin, J. H. James, S. N. Joffe, A. E. A. Kulneff-Herlin, and J. E. Fischer. Effect of jejunoileal bypass on plasma and brain amino acids in the rat. Journal of Neurochemistry, 38(4):1170-1173, 1982.

[28] A. T. B. Moir and D. Eccleston. The effects of precursor loading in the cerebral metabolism of 5-hydroxyindoles. Journal of Neurochemistry, 15:1093-1108, 1968.

[29] M. D. Diksic, S. Nagahiro, T. Chaly, T. L. Sourkes, Y. L. Yamamoto, and Feindel. W. Serotonin synthesis rate measured in living dog brain by positron emission tomography. Journal of Neurochemistry, 56(1):153-162, 1991.

[30] J. Korf, H. M. Van Praag, and J. B. Sebens. Serum tryptophan decreased, brain tryptophan increased and brain serotonin unchanged after probenecid loading. Brain Research, 42:239-242, 1972.

[31] D. Burns, J. London, D. J. Brunswick, M. Pring, D. Garfinkel, J. L. Rabinowitz, and J. Mendels. A kinetic analysis of 5-hydroxyindoleacetic acid excretion from rat brain and csf. Biological Psychiatry, 11(2):125-157, 1976.

[32] B. K. Madras and T. L. Sourkes. Metabolism of $\alpha$-methyl-tryptophan. Biochemical Pharmacology, 14:1499-1506, 1965.

[33] T. W. Stone and J. H. Connick. Quinolinic acid and other kynurenines in the central nervous system. Neuroscience, 15(3):597-617, 1985. 
[34] M. Diksic. unpublished data.

[35] T. N. Tozer, N. H. Neff, and B. B. Brodie. Application of steady-state kinetics to the synthesis rate and turnover time of serotonin in the rat brain of normal and reserpine-treated rats. Journal of Pharmacology and Experimental Therapeutics, $153(2): 177-182,1966$.

[36] N. B. Neff, T. N. Tozer, and B. B. Brodie. Application of steady-state kinetics to studies of the transfer of 5-hydroxyindoleacetic acid from brain to plasma. Journal of Pharmacology and Experimental Therapeutics, 158(2):214-218, 1967.

[37] R. C. Lin, E Costa, N. E. Neff, C. T. Wang, and S. H. Ngai. In vivo measurement of 5-hydroxytryptamine turnover rate in the rat brain from the conversion of ${ }^{14} \mathrm{C}$-tryptophan to ${ }^{14} \mathrm{C}$-5-hydroxytryptamine. Journal of Pharmacology and Experimental Therapeutics, 170(2):232-238, 1969.

[38] E. Costa and N. H. Neff. Estimation of turnover rates to study the metabolic regulation of the steady-state level of monoamines. In A. Lajtha, editor, Handbook of Neurochemistry, Volume 4, chapter 3, pages 45-90. Plenum Press, New York, 1970.

[39] J. D. Lane, C. T. Co, and J. E. Smith. Determination of simultaneous turnover of serotonin, dopamine and norepinephrine in the telencephalon of unrestrained behaving rats. Life Sciences, 21:1101-1108, 1977.

[40] N. H. Neff, P. F. Spano, A. Groppetti, C. T. Wang, and E. Costa. A simple procedure for calculating the synthesis rate of norepinephrine, dopamine and serotonin in rat brain. Journal of Pharmacology and Experimental Therapeutics, 176(2):701-710, 1971. 
[41] P. Tracqui, P. Brezillon, J. F. Staub, Y. Morot-Gaudry, M. Hamon, and A. M. Perault-Staub. Model of brain serotonin metabolism I. Structure determination - parameter estimation. American Journal of Physiology, 244:R193-R205, 1983.

[42] P. Tracqui, P. Brezillon, J. F. Staub, Y. Morot-Gaudry, M. Hamon, and A. M. Perault-Staub. Model of brain serotonin metabolism II. Physiological interpretation. American Journal of Physiology, 244:R206-R215, 1983.

[43] T.L. Sourkes. Alpha-methyltryptophan and its actions on tryptophan metabolism. Fed. Proc., 30:897-903, 1971.

[44] C.S. Patlak, R.G. Blasberg, and J.D. Fenstermacher. Graphical evaluation of blood-to-brain transfer constants from multiple-time uptake data. Journal of Cerebral Blood Flow and Metabolism, 3:1-7, 1983.

[45] A. Gjedde. High- and low-affinity transport of D-glucose from blood to brain. Journal of Neurochemistry, 36(4):1463-1471, 1981.

[46] M.E. Phelps, S.C. Huang, E.J. Hoffman, C. Selin, L. Sokoloff, and D.E. Kuhl. Tomographic measurement of local cerebral glucose metabolic rate in humans with (F-18)-2-fluoro-2-deoxy-D-glucose: validation of method. Ann. Neurol., 6:371-388, 1979 .

[47] Fernstrom J.D., M.J. Hirsch, and Faller D.V. Tryptophan concentrations in rat brain; failure to correlate with free serum tryptophan or its ratio to the sum of other serum neutral amino acids. Biochem. J., 160:589-595, 1976.

[48] Philip R. Bevington. Data Reduction and Error Analysis for the Physical Sciences. McGraw-Hill, New York, 1969. 
[49] J.L. Venero, A. Machado, and J. Cano. Turnover of dopamine and serotonin and their metabolites in the striatum of aged rats. Journal of Neurochemistry, 56:1940-1948, 1991.

[50] R. V. Stineman. Constantly well-behaved method of interpolation. Creative Computing, 6:54-57, 1980.

[51] N. R. Draper and H. Smith. Applied Regression Analysis. Wiley, New York, 1966.

[52] Robert R. Sokal and F. James Rohlf. Biometry. W. H. Freeman and Company, New York, 2nd edition, 1981.

[53] N. Baber, L. Halliday, L. Sibeon, and O. Littlert. The interaction between indomethacin and probenecid. Clin. Pharmacol. Therapeutics, 24:298-307, 1978.

[54] J. W. Paxton. Interaction of probenecid with the protein binding of methotrexate. Pharmacology, 28:86-89, 1984.

[55] Patrick J. McNamara, Vreny Trueb, and Klaus Stoekel. Ceftriaxone binding to human serum albumin. Biochemical Pharmacology, 40(6):1247-1253, 1990.

[56] J. Korf. Turnover rate assessments of cerebral neurotransmitter amines and acetylcholine. In A. A. Boulton, G. B. Baker, and J. M. Baker, editors, Neuromethods, vol.2: Amines and their metabolites., pages 407-456. Humana Press, Clifton, New Jersey., 1985.

[57] J. N. Davis, A. Carlsson, V. McMillan, and B. K. Siesjo. Brain tryptophan hydroxylation: dependence on arterial oxygen tension. Science, 182:72-74, 1973. 
[58] M. Kirikae, M. Diksic, and Y. L. Yamamoto. The transfer coefficients for L-valine and the rate of incorporation of $\mathrm{L}-\left[{ }^{14} \mathrm{C}\right]$-valine into proteins in normal adult rat brain. Journal of Cerebral Blood Flow and Metabolism, 8:598-605, 1988.

[59] Carolyn B. Smith. The measurement of regional rates of cerebral protein synthesis in vivo. Neurochemical Research, 16(9):1037-1045, 1991.

[60] P. Lestage, M. Gonon, P. Lepetit, P. A. Vitte, G. Debilly, C. Rossatto, D. Lecestre, and P. Bobilier. An in vivo kinetic model with $\mathrm{L}-\left[{ }^{35} \mathrm{~S}\right]$ methionine for the determination of local cerebral rates for methionine incorporation into protein in the rat. Jounal of Neurochemistry, 48:352-363, 1987.

[61] R. E. Keen, J. R. Barrio, S.-C. Huang, R. A. Hawkins, and M. E. Phelps. In vivo cerebral protein synthesis rates with leucyl-transfer RNA used as a precursor pool: Determination of biochemical parameters to structure kinetic models for positron emission tomography. Journal of Cerebral Blood Flow and Metabolism, 9:429-445, 1989. 